\title{
Phonons in models for icosahedral quasicrystals : low frequency behaviour and inelastic scattering properties
}

\author{
J. Los ('), T. Janssen (') and F. Gähler ( $\left.{ }^{2}\right)$ \\ (') Institute for Theoretical Physics, University of Nijmegen, $6525 \mathrm{ED}$ Nijmegen, \\ The Netherlands \\ ( $\left.{ }^{2}\right)$ Département de Physique Théorique, Université de Genève, 24 quai Ernest Ansermet, \\ $\mathrm{CH}-1211$ Genève 4 , Switzerland
}

(Recened 14 January 1993, accepted in final form 18 February 1993)

\begin{abstract}
A detailed study of the low frequency behaviour of the phonon spectrum for 3dimensional tiling models of icosahedral quasicrystals is presented, in commensurate approximations with up to 10336 atoms per unit cell. The scaling behaviour of the lowest phonon branches shows that the widths of the gaps relative to the bandwidths vanish in the low frequency limit. The density of states at low frequencies is calculated by Brillouin zone integration, using either local linear or local quadratic interpolation of the branch surface. For perfect approximants it appears that there is a deviation from the normal $\omega^{2}$-behaviour already at relatively low frequencies, in the form of pseudogaps. Also randomized approximants are considered, and it turns out that the pseudogaps in the density of states are flattened by randomization. When approaching the quasiperiodic limit, the dispersion of the acoustic branches becomes more and more isotropic, and the two transversal sound velocities tend to the same value. The dynamical structure factor is. determined for several approximants, and it is shown that the linearity and the isotropy of the dispersion are extended far beyond the range of the acoustic branches inside the Brillouin zone. A sharply peaked response is observed at low frequencies, and broadening at higher frequencies. To obtain these results, an efficient algorithm based on Lanczos tridiagonalisation is used.
\end{abstract}

\section{Introduction.}

In recent work on the lattice dynamics of 3-dimensional icosahedral Penrose tilings (i-PT), which are models for icosahedral quasicrystals, the total vibrational density of states (DOS), the scaling behaviour of the spectrum and the character of the eigenvectors have been considered [1]. This was done for perfect, symmetrized and randomized periodic approximants of the i-PT. In the present paper, we consider exactly the same models again, but concentrate this time on the details of the low frequency behaviour. In reference [1] the structure of the i-PT and the various tilings related to it are explained in detail, so that in the present paper we can restrict ourselves to a brief description of the structure of the tilings and the dynamical model.

The 3-dimensional icosahedral Penrose tiling (i-PT) is a space filling of 3-dimensional physical space with two kinds of rhombohedra, a thick and a thin one. The thick rhombohedron 
occurs $\phi$ times more frequently than the thin one, where $\phi=(\sqrt{5}+1) / 2$ the golden number. The i-PT has no 3-dimensional lattice periodicity, but is quasiperiodic. The vertices of the i-PT can be found by taking the intersection of a hypercubic lattice periodic structure in 6 dimensions with the 3-dimensional physical space, which appears as a subspace of the 6dimensional space. The positions of the vertices of the tiling are integer linear combinations of six rationally independent vectors, pointing to the corners of an icosahedron. The commensurate approximations (or approximants) are approximations of the i-PT which have 3dimensional lattice periodicity, and can be obtained as the intersection of a deformed 6dimensional structure with physical space. There is a whole series of approximants [2], with various kinds of unit cells. In this paper, we will only use approximants which have cubic lattice periodicity. These approximants are commonly labelled by the rational substitute that is used for the golden number $\phi$ occurring in the 6-dimensional lattice basis, i.e. $\phi=$ $1 / 1-, 2 / 1-, 3 / 2-, 5 / 3-, 8 / 5-\ldots$ approximants, having respectively $32,136,576,2440$ and 10336 vertices inside their cubic unit cell. These approximants have almost $T_{h}$ point group symmetry. By adding relatively few sites, the $T_{h}$ symmetry can be made complete, and one obtains the so-called symmetrized approximants. The randomized approximants are obtained from a perfect approximant by a process of repeatedly moving certain atomic positions within the unit cell, in such a way that the structure is still space filling with the two rhombohedra mentioned above. This kind of randomization has been studied by Tang [3].

A dynamical model based on these tilings is obtained by placing an atom on each vertex and putting springs between neighbouring atoms at distances equal to $2, \sqrt{2+\phi}(\approx 1.902)$ or $\sqrt{6-3 \phi}(\approx 1.070)$, which are respectively the face diagonals and edges of the rhombohedra, and the short body diagonal of the thin rhombohedron. For the symmetrized approximants one extra short distance occurs, namely $2 \phi-2(\approx 1.236)$. For simplicity, the mass of all atoms was taken equal to 1 . For most of the results presented in this paper the strengths of all springs were taken to be equal. In order to make a correct quantitative comparison of the results with similar results for a bcc-lattice having one atom per primitive unit cell connected to 14 neighbours by equal springs with spring constant 1, we have taken the strength $\alpha$ of the springs such that $\alpha \gamma$ is equal to 14 , where $\gamma$ is the mean number of neighbours per atom ( $\approx 13.2$ for the perfect approximants).

For the i-PT the system of masses and springs is quasiperiodic and gives rise to an infinite dynamical matrix. However, the problem can be made finite by using a cluster approximation (with free or clamped boundary conditions) or a commensurate approximation. In the latter case the dynamical matrix depends on a wave vector $\mathbf{k}$, and to find all states we have to solve the eigenvalue/eigenvector problem for all wave vectors in the first Brillouin zone. In a previous paper [4] we have presented the total density of states for clusters of about 16000 atoms. In the present paper we are interested in the low frequency behaviour of the density of states. To investigate this for the infinite tiling, the cluster approximation seems to be less adapted, because one will always find a finite number of discrete eigenstates, so that there will be a gap between the modes at frequency zero and the next lowest modes. One would have to consider very large clusters to observe convergence of the density of states at low frequency under enlargement of the system. Therefore, in the present paper we use systematic commensurate approximants to understand the scaling behaviour of the band structure at low frequency.

A 1-dimensional example of a quasicrystal is the Fibonacci chain. It consists of a welldefined quasiperiodic sequence of long and short intervals. A simple dynamical model for this chain is obtained by putting atoms on its vertices and springs between each neighbouring pair, with spring constants $K$ and $K^{\prime}$ for, respectively, a long and a short interval. Just as for the i-PT there exists an infinite systematic sequence of periodic approximants, converging to the 
quasiperiodic chain. For successive approximants the number of atoms per unit cell grows with a factor $\phi$ (i.e. golden number), and so does the number of branches in the phonon spectrum for these approximants. Labelling the approximants by an integer $n$, the $n$-th approximant has approximately $C \phi^{n}$ atoms per unit cell, where $C$ is some constant. An important result for the behaviour of the band structure at low frequencies, obtained by Kohmoto [5], is the following :

$$
\lim _{n \rightarrow \infty} \frac{g_{1}(n)}{\omega_{i}(n)}=0
$$

where $g_{i}(n)$ is the width of the $i$-th gap from below in the $n$-th approximant, and $\omega_{1}(n)$ the frequency level in the middle of this gap. This result, which was presented in a slightly different way in [5], was obtained analytically by using the transfer matrix method, which can only be applied for 1-dimensional systems. It means that the widths of the gaps relative to the bandwidths vanish in the limit of $\omega \rightarrow 0$, and consequently the bending of the dispersion curve at low frequencies, near the center and the boundary of the Brillouin zone, i.e. near the gaps, is restricted to a relatively narrow region in $k$-space. Therefore the deviation from the normal constant behaviour of the DOS at low frequencies, which holds for 1dimensional periodic structures due to the linear dispersion $\omega=c k$, will be restricted to a correspondingly small interval in frequency space, so that it will not be visible, unless one uses an integration method with a very high resolution. In fact, one could still speak of a linear dispersion at low frequencies in the incommensurate limit, although it is interrupted by an infinite number of relatively small gaps.

In three dimensions, things are considerably more complicated, even for models where all spring constants are the same, which would make the 1-dimensional problem trivial. Analytic methods, which work well for 1-dimensional systems, such as the transfer matrix formalism, fail for 3-dimensional systems. It is also more difficult to acquire a qualitative understanding of the spectrum. A 1-dimensional quasiperiodic sequence can always be considered as an incommensurately modulated periodic sequence. The degenerate perturbation theory then permits us to estimate where gaps will occur and how large they are in first order. A 3dimensional icosahedral quasicrystal cannot be obtained by perturbing some periodic structure.

Experimentally the phonon properties of icosahedral quasicrystals have been studied by inelastic neutron scattering measurements. The results of all these experiments indicate the existence of well-defined propagating modes with isotropic linear dispersion at low frequency, both for transversal and longitudinal modes [6-8]. In the present paper we show the results of calculations of the dynamical response to inelastic neutron scattering by considering a function which contains the most significant part of the dynamical structure factor. These calculations are done using either a direct method, which requires the determination of all eigenvalues and eigenvectors of the dynamical matrix, or a recursion method [9]. Concerning the latter method we introduce a technique to obtain the relevant information from the recursion coefficients, which is different from other methods used in the literature and which may be more accurate.

The commensurate 8/5-approximant has a cubic unit cell with 10336 atoms. This means that the order of the dynamical matrix, for which we have to find the eigenvalues, is equal to 31008 . For our computer system there is no way to diagonalize such large matrices by direct methods, not only for storage capacity reasons, but also for computation time reasons. The computation time needed for the diagonalization of a matrix is proportional to the third power of the order of the matrix ! At present our limit is more or less reached with matrices of order of a few thousand. However, in our model tilings each atom interacts only with a limited number of neighbours, and therefore the dynamical matrix is very sparse, especially for the approximants with large unit cells. For example, in the 8/5-approximation only about $0.1 \%$ of the matrix-elements are nonzero. The sparseness of the dynamical matrix further increases for 
the higher approximants, when the number of atoms $N$ per unit cell becomes larger, since the total number of matrix-elements is equal to $9 N^{2}$ whereas the maximal number of nonzero elements is only $9(\gamma+1) N$, where $\gamma$ is the mean number of interacting neighbours per atom $(\approx 13.2$ in our models). The fraction of nonzero elements is thus maximally equal to $(y+1) / N$. In addition, part of this fraction may also be zero due to the relative orientation of the interacting atoms. Another useful property is that the matrices are hermitian, which means that only the lower (or upper) triangular matrix has to be stored. Finally, a lot of matrix elements are equal, due to the limited number of neighbour configurations occurring in the model. For the perfect 8/5-approximant there are only 425 different matrix elements. Such a matrix can be stored very economically by using 3 integer arrays and one real array containing the 425 reals. One integer array of length $3 N$, which is the order of the matrix, contains the number of nonzero elements of the $3 N$ rows of the lower triangular part of the matrix. The other two integer arrays are respectively for indicating in which column each of the nonzero elements occurs and which value it has, indicated by an integer pointing to one of the 425 reals.

Until now there is no direct diagonalization method which makes efficient use of the sparseness. To take advantage of the sparseness of the matrices one has to think about iterative methods, in particular methods which require only matrix-vector multiplications. Such a method is the Lanczos algorithm. In this method, discovered by Lanczos in 1950, the matrix is transformed to tridiagonal form. However, the method is numerically unstable, due to rounding errors. This was already known to Lanczos, and since 1950 the algorithm was intensively studied by various people in order to find out how it could still be of use for the determination of eigenvalues (and eigenvectors), in spite of its deficiencies [10-13]. For a long time it was believed that the simple algorithm, without very expensive modifications, could only be used to compute some of the extremal eigenvalues. There are now, however, practical Lanczos procedures without such expensive modifications, denoted as « Lanczos algorithms with no reorthogonalization », which are used to find all distinct eigenvalues and corresponding eigenvectors of large hermitian matrices. One such procedure has been developed by Cullum and Willoughby and described in reference [12]. The method can be used very well to find all eigenvalues within a certain interval, and this is precisely what we needed in our study of the low frequency behaviour. The algorithm which we constructed to find a certain number of the lowest eigenvalues of the very large sparse matrices is completely based on the « Lanczos algorithm with no reorthogonalization ».

The outline of the paper is as follows. In section 2 we present the phonon band structure at low frequency and study its scaling behaviour. In section 3 the DOS at low frequencies for perfect and randomized approximants is presented. The DOS is obtained by a Brillouin zone integration method, which is described in appendix B. Calculations of the dynamical response to inelastic neutron scattering are presented in section 4 for various approximants. Appendix A contains a description of the Lanczos algorithm, with its features and deficiencies.

\section{Band structure at low frequency.}

The scaling behaviour of the phonon band structure at low frequency can be studied directly by displaying the dispersion curves for successive approximants along a path in the Brillouin zone. First we define some special points inside the Brillouin zone :

$$
\begin{array}{lll}
\Gamma=(0,0,0) & \mathrm{X}_{1}=\pi / a(1,0,0) & \mathrm{M}_{1}=\pi / a(1,1,0) \\
\mathrm{R}=\pi / a(1,1,1) & \mathrm{X}_{2}=\pi / a(0,1,0) & \mathrm{M}_{2}=\pi / a(1,0,1) \\
& \mathrm{X}_{3}=\pi / a(0,0,1) & \mathrm{M}_{3}=\pi / a(0,1,1)
\end{array}
$$


where $a$ is the lattice constant for an i-PT approximant, which we define as :

$$
a=(N / \rho)^{1 / 3},
$$

where $N$ is the number of atoms per unit cell and $\rho$ the atomic density, which we assume to be equal to 1 for all structures considered in this paper. At each step to a larger approximant the lattice constant $a$ increases approximately by a factor $\phi$, so that the number of atoms per unit cell increases by a factor $\phi^{3}$.

The path we have chosen for displaying the dispersion is : $\Gamma \rightarrow \mathrm{X}_{1} \rightarrow \mathrm{M}_{1} \rightarrow \Gamma \rightarrow \mathrm{R} \rightarrow$ $\mathrm{M}_{3} \rightarrow \mathrm{X}_{2} \rightarrow \Gamma$. The lowest 24 branches along this path are shown in figures $1 \mathrm{a}, 1 \mathrm{~b}$ and $1 \mathrm{c}$, for respectively the $2 / 1-, 3 / 2-$ and 8/5-approximant, which have respectively 136,576 and 10336 atoms in their cubic unit cell. Firstly, we observe that these pictures are globally very similar. Note, however, that the scale on the frequency axis changes at each step to a larger approximant. A second important feature is that the widths of the gaps in the dispersion relative to the bandwidths decrease at each step. For example, the gap at about $\omega=0.8$ occurring at $X_{1}$ for the 2/1-approximant scales to the much smaller gap at about $\omega=0.5$ at the same k-point for the 3/2-approximant. In the latter case the gap is hardly visible, because it is so small. Especially for the 3/2- and 8/5-approximant, it happens that eigenfrequencies are so close that they are not distinguishable in the pictures of figure 1 . For example, two of the three acoustic branches are almost degenerate (also for the 2/1-approximant). These two correspond to the transversal modes. The fact that the gaps vanish in the low frequency limit implies that the scaling behaviour of a number of the lowest branches will become more and more exact when tending to the quasiperiodic limit, i.e. at each step the same band structure will show up, scaled by a factor $\phi$ along the frequency axis.

In the quasiperiodic limit, due to the (almost) icosahedral symmetry, not only the dispersion curves that correspond to the transversal modes are expected to become equal, but it is also expected that the dispersion is isotropic at low frequencies, i.e. the sound velocity is isotropic. In the low frequency limit the acoustic branches behave linearly in $k$ :

$$
\omega_{s}(\mathbf{k})=c_{s}(\phi, \theta) k
$$

where $s=1,2,3$ for the three acoustic branches. We have checked the isotropy of the dispersion and the degeneracy of both transversal sound velocities $c_{1}$ and $c_{2}$ numerically for a number of approximants, by computing the lowest three eigenfrequencies for a discrete set of directions in k-space at distance $\pi /(10 a)$ from the origin. From these eigenfrequencies a corresponding set of numbers $c_{s}\left(\phi_{j}, \theta_{j}\right)$ can be determined, and from these the mean value $\left\langle c_{s}\right\rangle$ and the standard deviation $\Delta c_{s}$, defined for each of the three acoustic branches by:

$$
\begin{aligned}
\left\langle c_{s}\right\rangle & =\frac{1}{M} \sum_{j=1}^{M} c_{s}\left(\phi_{j}, \theta_{j}\right) \\
\Delta c_{s} & =\sqrt{\left\langle c_{s}^{2}\right\rangle-\left\langle c_{s}\right\rangle^{2}}
\end{aligned}
$$

where $\left\langle c_{s}^{2}\right\rangle$ is the mean value of $c_{s}^{2}$ and $M$ the number of $\mathbf{k}$-directions being considered. The results are listed in table $I$. The numbers in this table have to be multiplied by a factor $10^{-1}$ to obtain agreement with our choice of the spring constant.

Table I shows clearly the tendency to isotropy and the degeneracy of the transversal sound velocities. It is remarkable, that this is emphasized even more by the results for the $5 / 3$ approximant than for the 8/5-approximant. Probably this is due to the additional bcc lattice symmetry, which is present only for the $p / q$-approximants with both $p$ and $q$ odd.

Since the widths of the gaps vanish in the low frequency limit, we expect that the band 


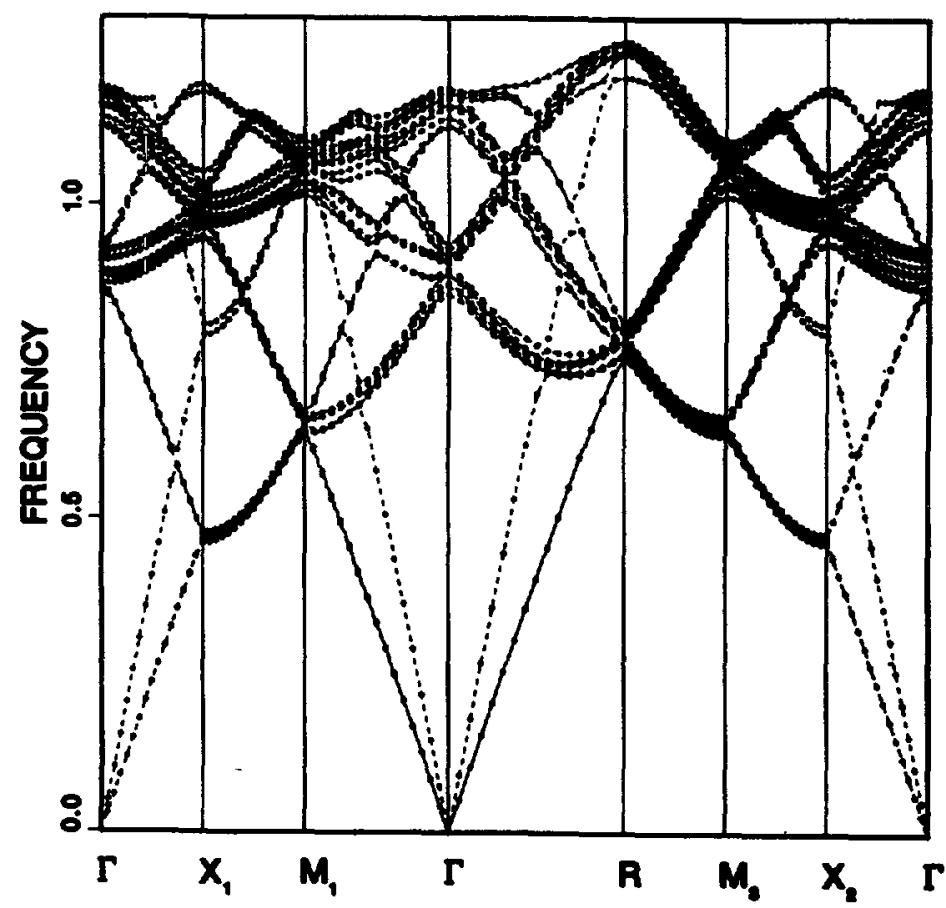

a)

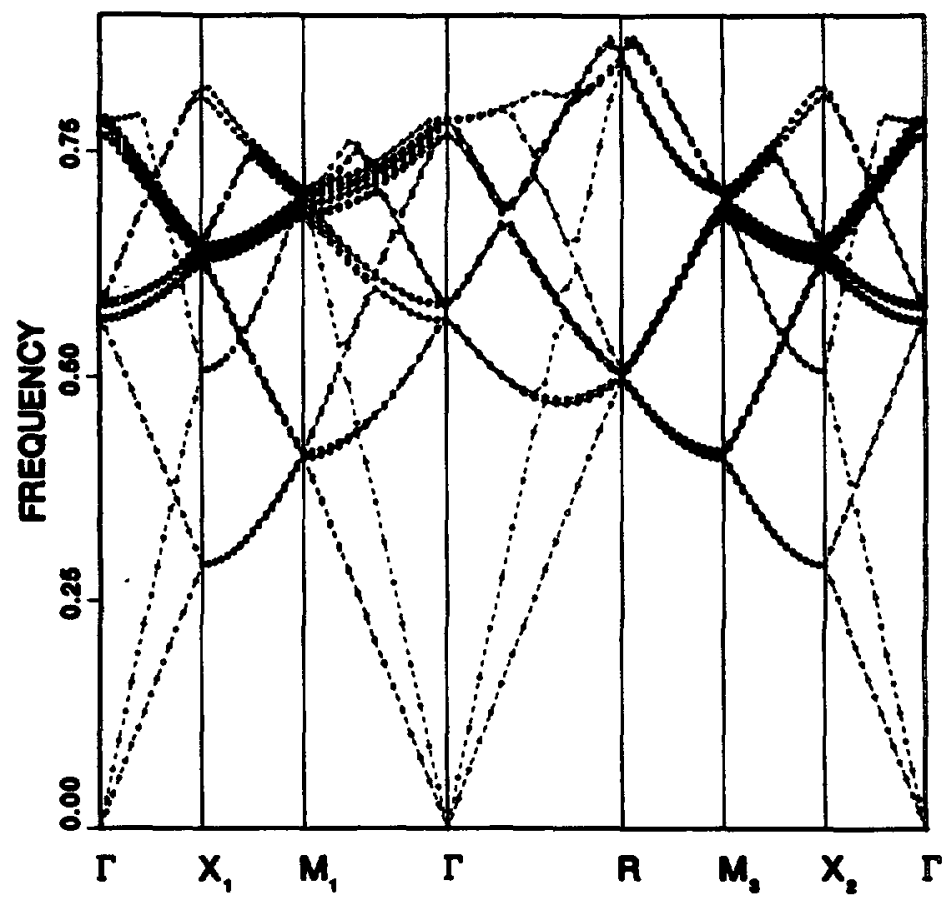

b)

Fig. 1. - a) The lowest 24 phonon branches for the $2 / 1$-approximant along a path through the Brillouin zone. b) As in figure $1 \mathrm{a}$, but for the 3/2-approximant. 


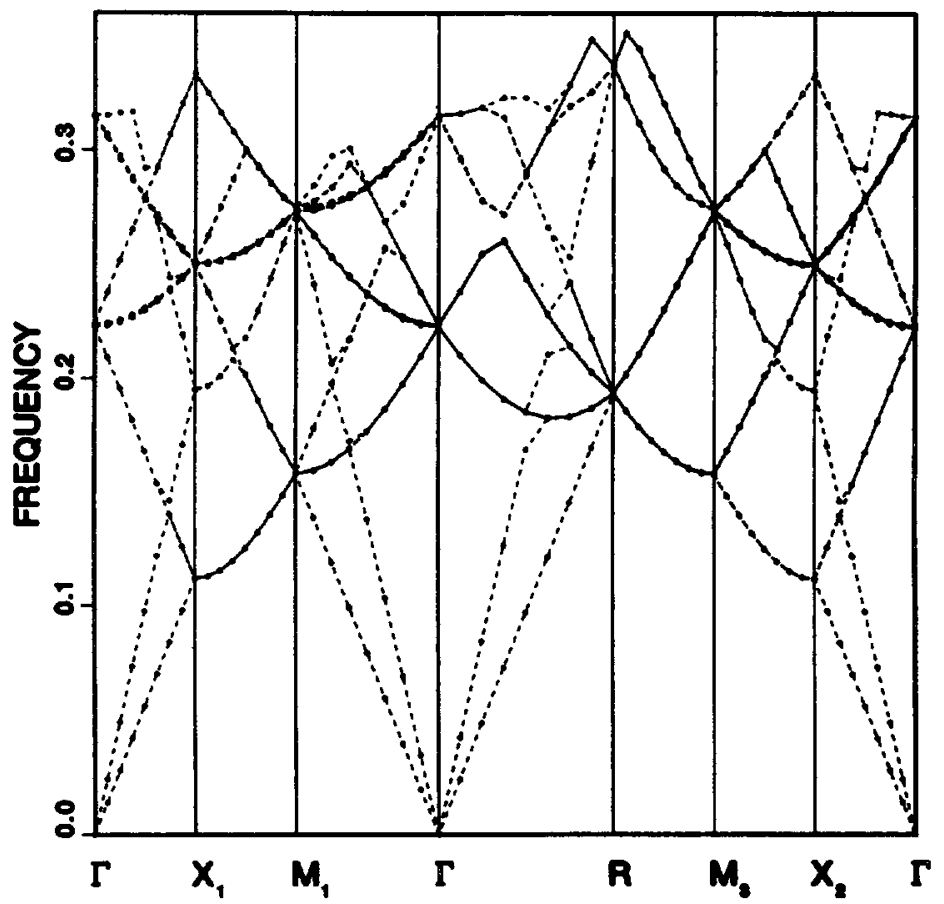

Fig. 1c. - As in figure 1a, but for the $8 / 5$-approximant.

Table I.

\begin{tabular}{|l|c|c|c|c|c|c|}
\hline & $\left\langle c_{1}\right\rangle$ & $\Delta c_{1}$ & $\left\langle c_{2}\right\rangle$ & $\Delta c_{2}$ & $\left\langle c_{3}\right\rangle$ & $\Delta c_{3}$ \\
\hline bcc & 7.4947 & 0.3289 & 8.2925 & 0.5032 & 13.8082 & 0.3725 \\
\hline 2/1-appr. & 7.7433 & 0.0255 & 7.7978 & 0.0211 & 13.5256 & 0.0417 \\
\hline 3/2-appr. & 7.7501 & 0.0177 & 7.7998 & 0.0294 & 13.5446 & 0.0197 \\
\hline 5/3-appr. & 7.7769 & 0.0007 & 7.7786 & 0.0006 & 13.5395 & 0.0009 \\
\hline 8/5-appr. & 7.7739 & 0.0026 & 7.7811 & 0.0043 & 13.5412 & 0.0031 \\
\hline
\end{tabular}

structure at low frequency tends to a normal linear behaviour, even far beyond the first zone in an extended zone scheme. Whether this is true can be checked by assuming a normal linear dispersion starting from the $\Gamma$-point, and then constructing from this the band structure in a reduced zone scheme by imposing the periodicity that corresponds to the periodicity of an i-PT approximant. By doing this for the 2/1- and 8/5-approximants we obtain figures $2 a$ and $2 b$, respectively. The proportionality constants $c_{s}(\phi, \theta)$ for the linear dispersion curves were taken to be equal to the true values for the corresponding approximant at each of the $k$ directions $(\phi, \theta)$ needed for the construction of the pictures. Note, however, that in particular 


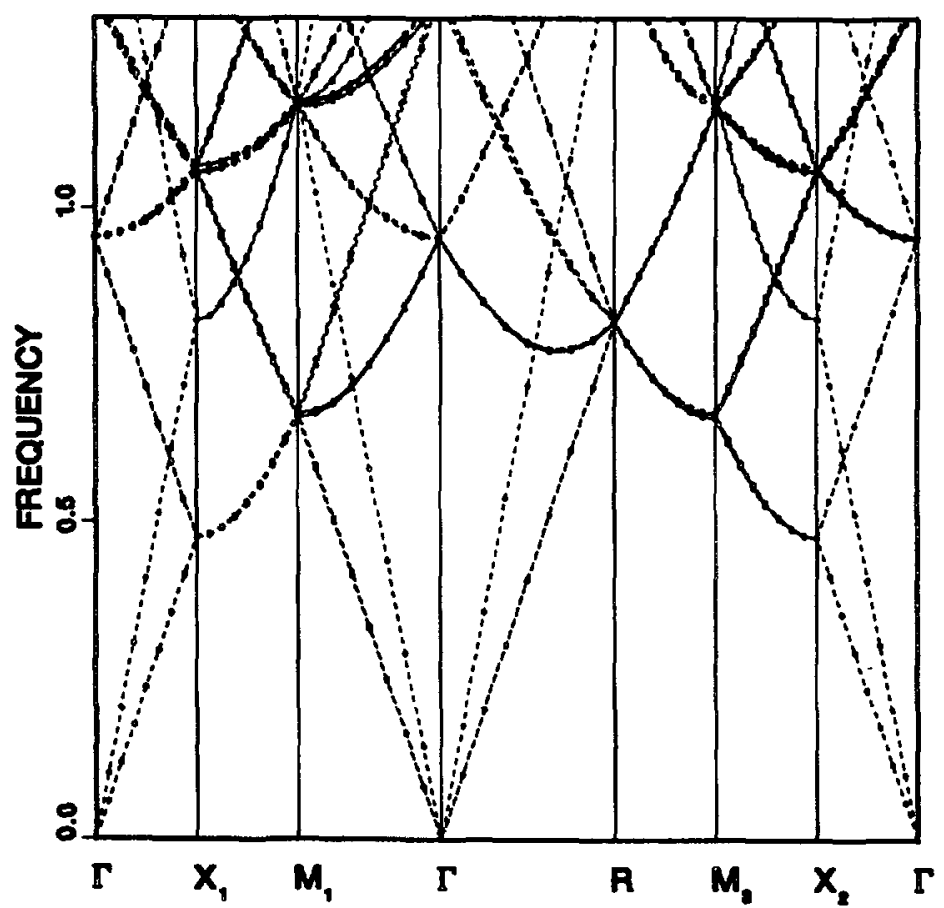

a)

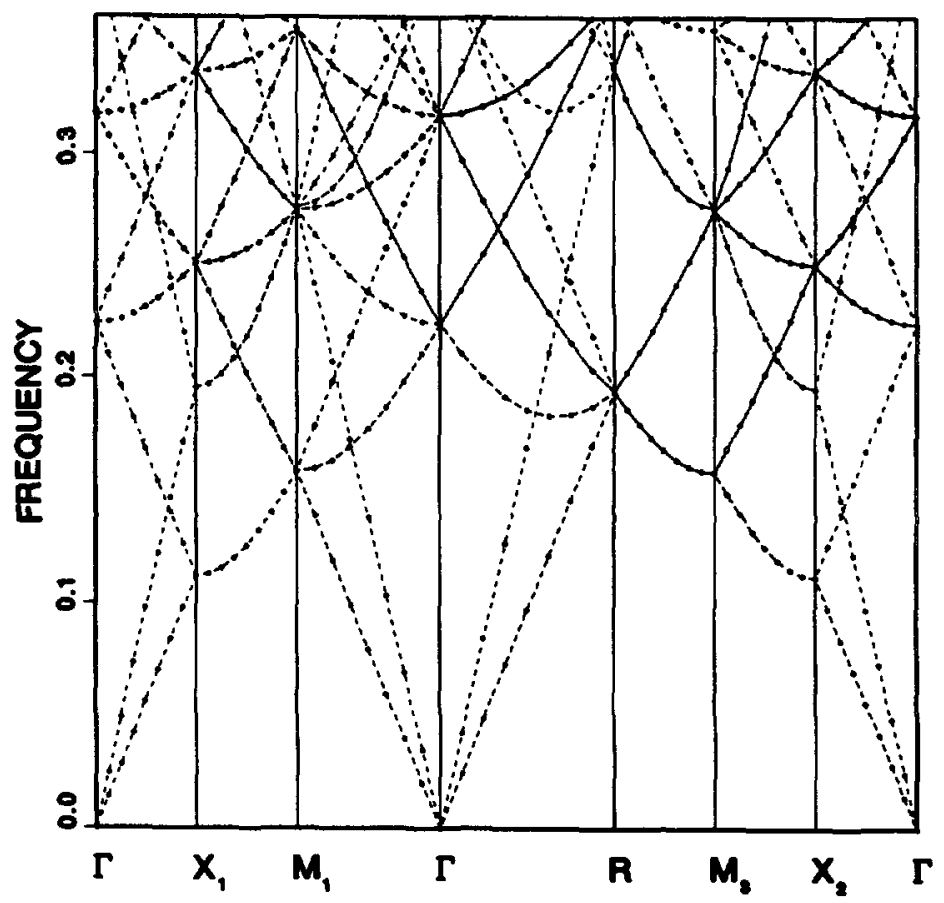

b)

Fig. 2. - a) Phonon band structure at low frequency for the $2 / 1$-approximant, obtained by assuming a linear dispersion in each direction, starting from the $\Gamma$-point in reciprocal space. b) As in figure $2 \mathrm{a}$, but for the 8/5-approximant. 
in the case of the 8/5-approximant we could as well have taken the mean values listed in table I, since the deviation from complete isotropy is already very small for this approximant. From figures $2 \mathrm{a}$ and $2 \mathrm{~b}$ one gets a very clear idea of what is happening. In fact, figures 1 and 2 show precisely to what extent the dispersion deviates from a normal linear behaviour. For the 2/1-approximant there is certainly some deviation in the frequency range of the lowest 24 branches (the total support of the spectrum is about [0,3.2], as can be seen be comparing the figures $1 \mathrm{a}$ and $2 \mathrm{a}$, but for the $8 / 5$-approximant the band structure shown in figure $1 \mathrm{c}$ is very close to that of figure $2 b$. For the latter case, the most important difference is that in figure $2 b$ the branches are crossing, whereas in figure 1c they do not cross. Note that in figure 1 the eigenfrequencies were connected in ascending order, since in the absence of any point group symmetry the branches are not supposed to cross each other. Therefore, the gaps in the dispersion for the 8/5-approximant along the path displayed in these figures will occur at all those $\mathbf{k}$-points where the branches cross in figure $2 \mathrm{~b}$. It is important to note that these gaps not only occur at the Brillouin zone centres and boundaries, due to the crossings of the branches coming from $\Gamma$-points lying on a line in k-space, but also at positions in the interior of the Brillouin zone, due to other branch crossings. In fact, these additional branch crossings seem to give rise to larger gaps than the crossings halfway between two $\Gamma$-points.

This behaviour can be illustrated more clearly by considering more branches. Figure $3 a$ shows the lowest 78 branches for the 3/2-approximant. Figure $3 \mathrm{~b}$ shows, for the same approximant, the same number of branches along the $(1,0,0)$-direction in a periodic zone scheme. In this picture we observe three linear dispersions starting from each of $\Gamma$-points, two of them being almost degenerate, so that they are hardly distinguishable in the picture. Following the linear dispersion curves from the $\Gamma$-points in figure $3 \mathrm{~b}$ we can easily find the positions where they cross other dispersion curves. These other dispersions are either linear dispersions starting from other $\Gamma$-points along the $(1,0,0)$-line, or dispersions stemming from $\Gamma$-points which do not lie on this line. One cannot expect that the polarizations of the modes corresponding to these latter dispersions are typically transversal or longitudinal with respect to the $(1,0,0)$-direction. On the other hand, it is possible that these modes are neither completely orthogonal to the ideal mode with a purely transversal or longitudinal polarisation relative to the $(1,0,0)$-direction. Therefore, in applying degenerate perturbation theory to estimate the size of the gaps, one may expect a coupling that is not very small, giving rise to a gap that is not very small either. Evidence that this is true may also be found in figure 1c. Whereas the gap in the «longitudinal dispersion » at the $X_{1}$-point at about $\omega=0.195$ is already so small that it is not visible any more in the picture, the gap occurring somewhat further along the same dispersion curve at about $\mathbf{k}=4 \pi / 5 a(1,0,0)$ between $\Gamma$ and $\mathrm{X}_{1}$ and at frequency $\omega=0.235$ is considerably larger.

\section{Density of states at low frequency.}

To obtain the total density of states (DOS) one has to integrate over all wave vectors inside the Brillouin zone :

$$
D(\omega)=\frac{1}{(2 \pi)^{3}} \sum \int_{\mathrm{BZ}} \mathrm{d} \mathbf{k} \delta\left(\omega-\omega_{s}(\mathbf{k})\right)
$$

where $\sum_{1}$ means summation over all branches. Due to the $\delta$-function this integral can be replaced by a surface integral :

$$
D(\omega)=\frac{1}{(2 \pi)^{3}} \sum_{s} \int_{S_{\omega}} \frac{\mathrm{d} S}{\left|\hat{\mathbf{n}} \cdot \nabla \omega_{\varepsilon}(\mathbf{k})\right|},
$$




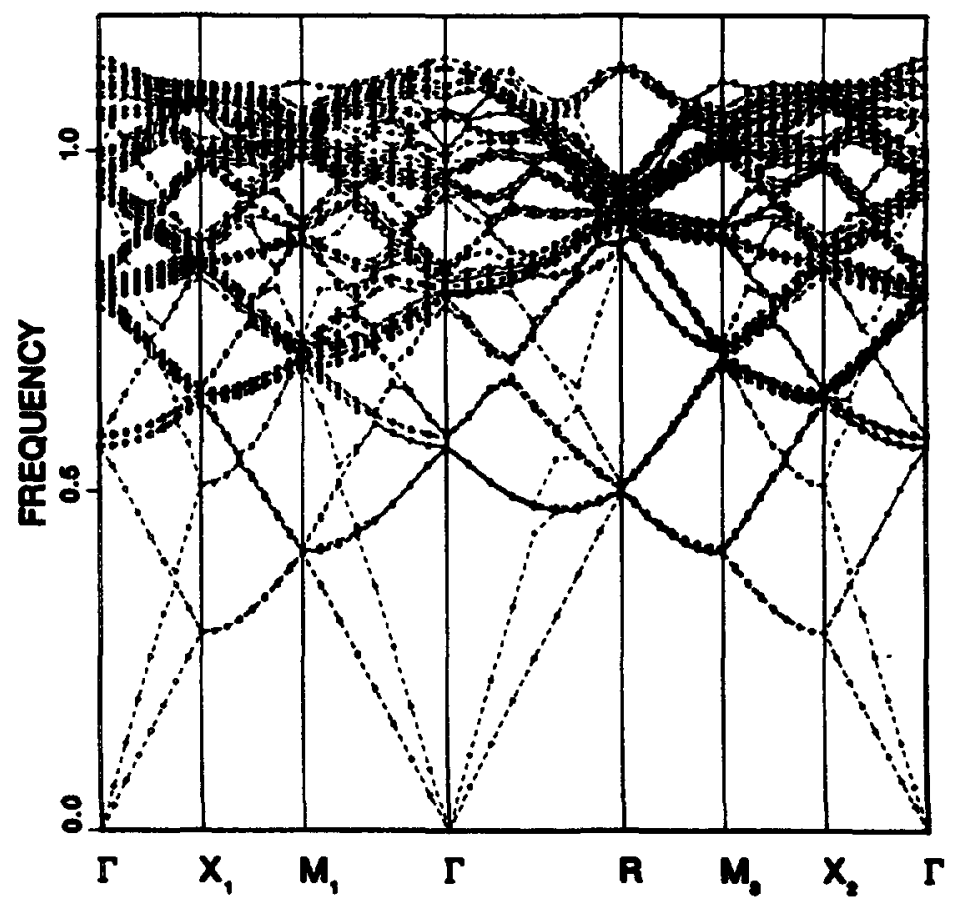

a)

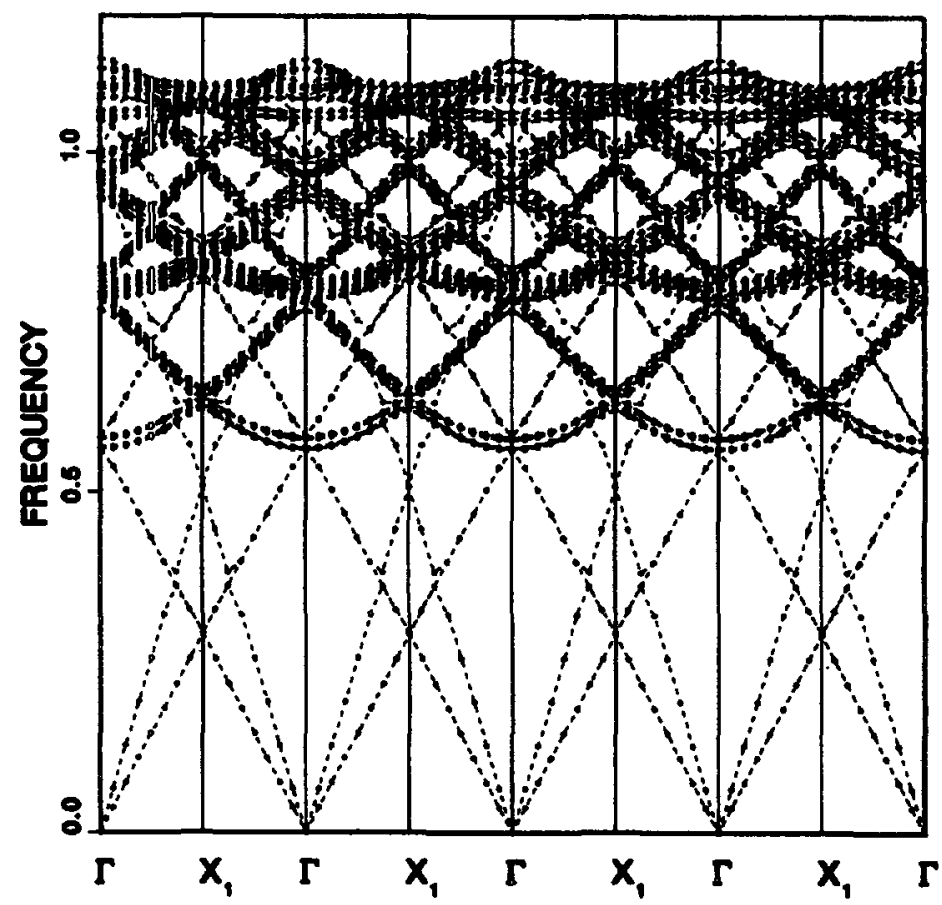

b)

Fig. 3. - a) The lowest 78 phonon branches for the 3/2-approximant along a path through the Brillouin zone. b) The lowest 24 phonon branches along the $(1,0,0)$-direction in a periodic zone scheme, for the 3/2-approximant. 
where $\int_{S_{\omega}}$ means integration over the surface of constant frequency and $\dot{n}$ denotes a unit vector orthogonal to the branch surface at wave vector $\mathbf{k}$. For the numerical evaluation of the DOS from (8) we used either a local linear or a local quadratic interpolation of the branch surface. Local quadratic interpolation was also used by MacDonald et al. [14] and by Methfessel [15]. The interpolated branch surfaces were fitted on a cubic grid of $k$-points inside the Brillouin zone. The eigenfrequencies at the corners of the cubes are prescribed by the eigenvalues of the dynamical matrix at this wave vector, which are determined by using a Lanczos algorithm with no reorthogonalization (see appendix A). Each of the small cubes can be divided into six equal tetrahedra, called simplices, of which the corners coincide with the corners of the cubes. Knowing the values on these corners one can perform a linear interpolation of the branch surface within the simplex. If, instead, we take eight small cubes forming one bigger cube and split this bigger cube up into six tetrahedra, one can perform a quadratic interpolation within each simplex, since now also the eigenfrequencies at the mid-edges of the tetrahedra are known. The density of the grid is indicated by an integer number $m$. For a grid $m$ interpolation the edge length of the small cube is equal to $\pi /(m a)$. We will use the linear interpolation besides the quadratic interpolation to serve as an indicator for the convergence of the DOS. Further details of the integration method are given in appendix $B$.

Due to the degeneracy of the eigenfrequencies at $k$ and $-k$ the Brillouin zone integration can be restricted to half of the total Brillouin zone, e.g. the part above the $k_{z}=0$ plane. However, to limit the amount computation time, we have restricted ourselves to only one octant of the cubic Brillouin zone. Because of the approximate tetrahedral symmetry present in the i-PT approximants we expect that the contributions from each of the four octants to the total DOS at low frequency are about the same. We have checked this approximation numerically for the 2/1-approximant, where it is clearly justified. For this approximant, figure 4 shows the contributions to the total DOS at low frequency from two octants using a grid 16 quadratic

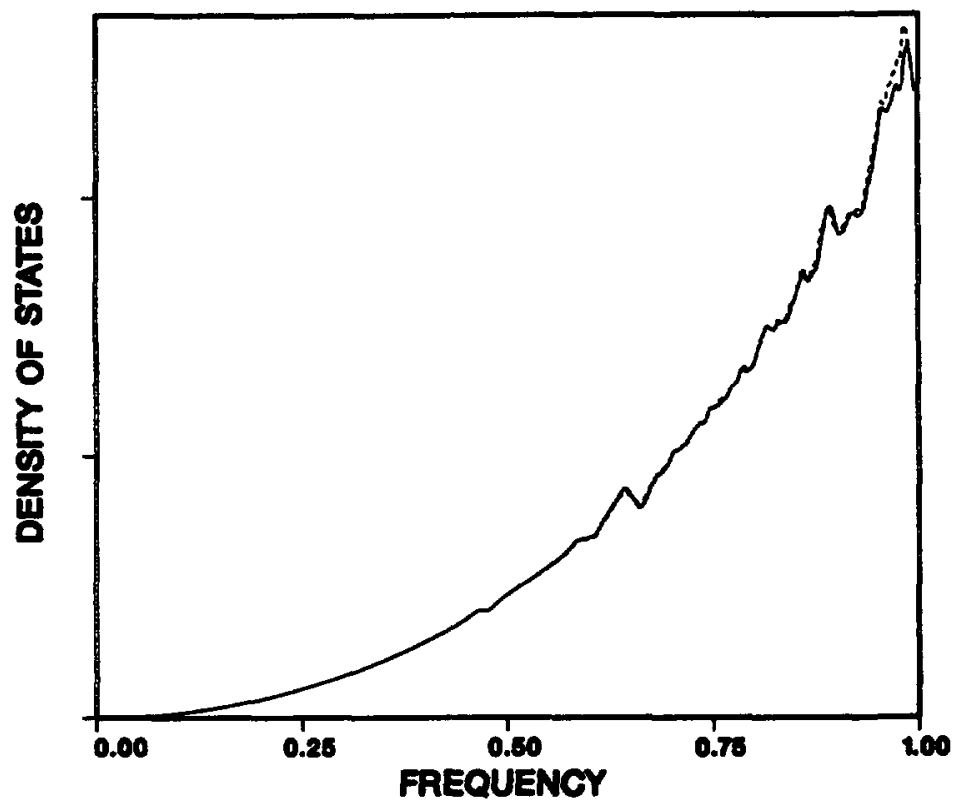

Fig. 4. - The contributions from two octants in the Brillouin zone to the total density of states at low frequencies for the 2/1-approximant, obtained from quadratic grid 16 interpolation. 


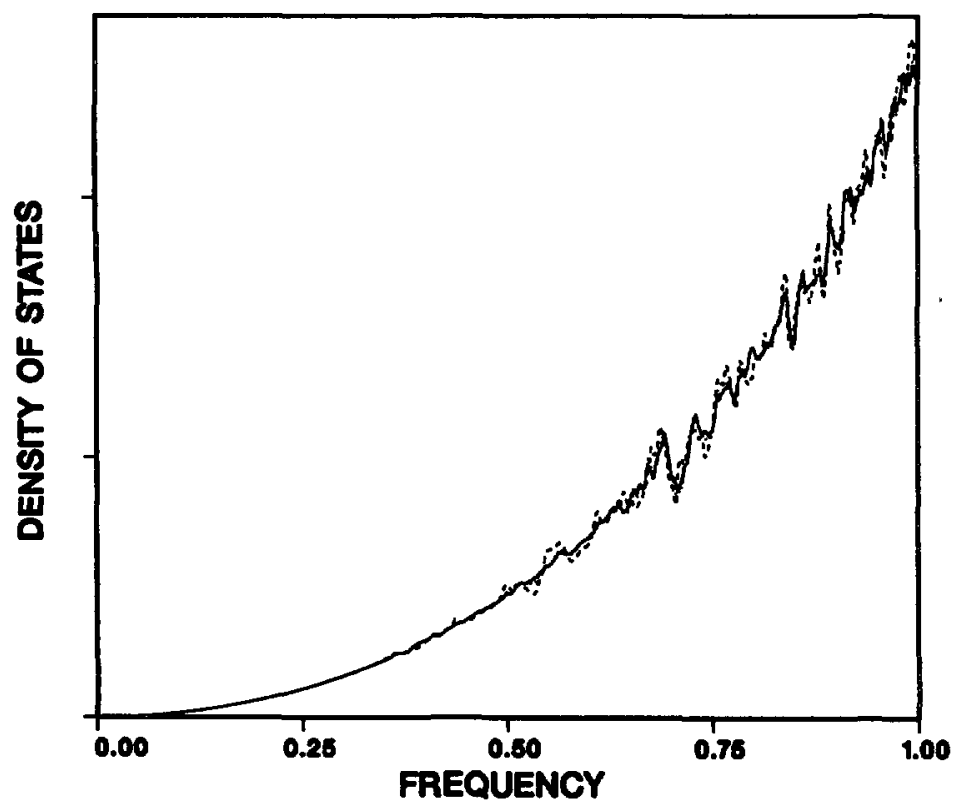

Fig. 5. - The normalized density of states at low frequency for the 3/2-approximant, obtained from quadratic grid 16 and grid 8 interpolation (solid and dashed line) and from linear grid 16 interpolation (dotted line). The dotted line is almost completely hidden behind the solid line, indicating the good convergence.

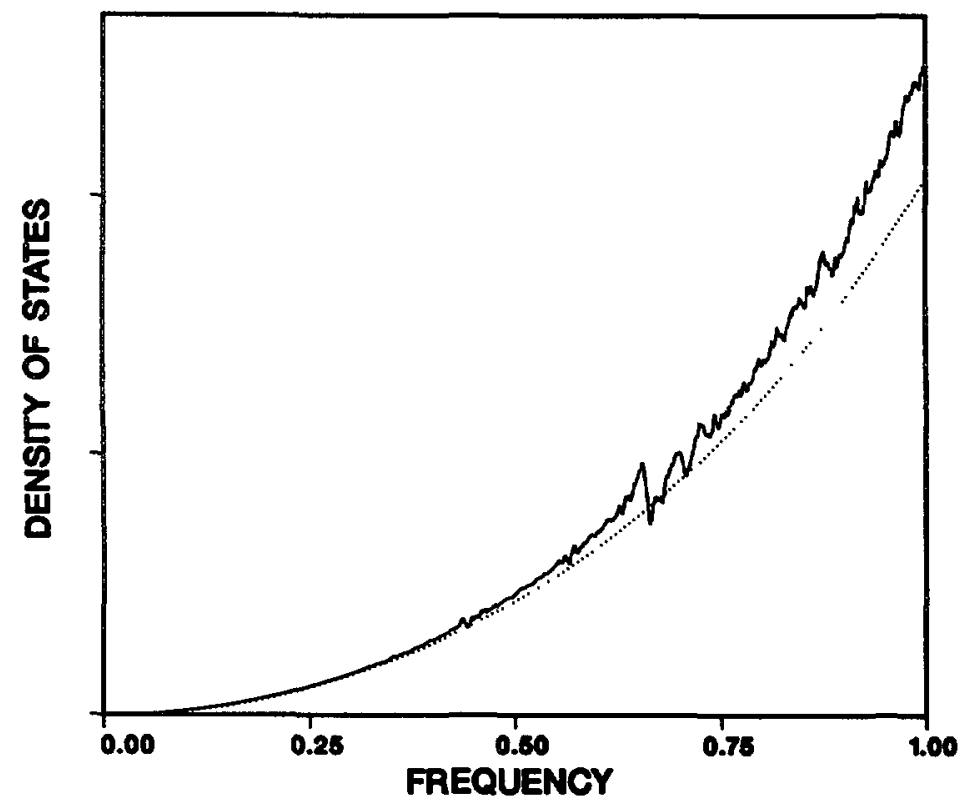

Fig. 6. - The normalized density of states at low frequency for the 5/3-approximant (solid line) and a bcc structure (dotted line) obtained from quadratic grid 16 interpolation. The linear grid 16 interpolation result (dashed line) is completely behind the quadratic interpolation result. 


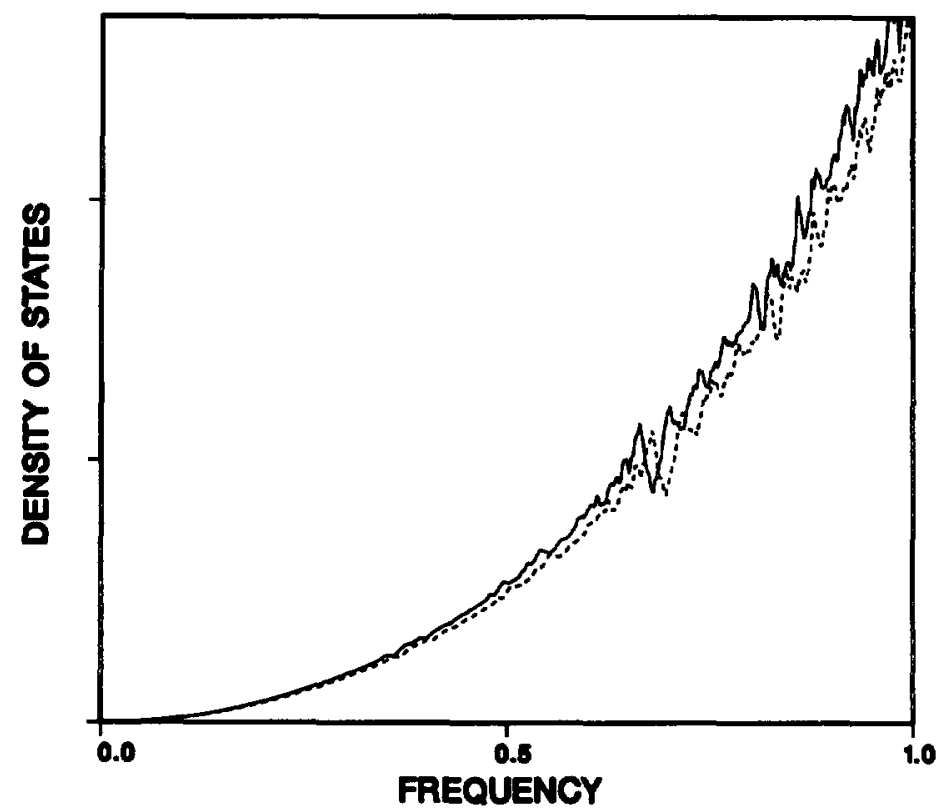

Fig. 7. - The normalized density of states at low frequency for the $3 / 2$-approximant, where the spring constant for the short bond length was taken two (dashed line) and three (dotted line) times as strong as the spring constant for the other bond lengths. Both result are obtained using quadratic grid 16 interpolation.

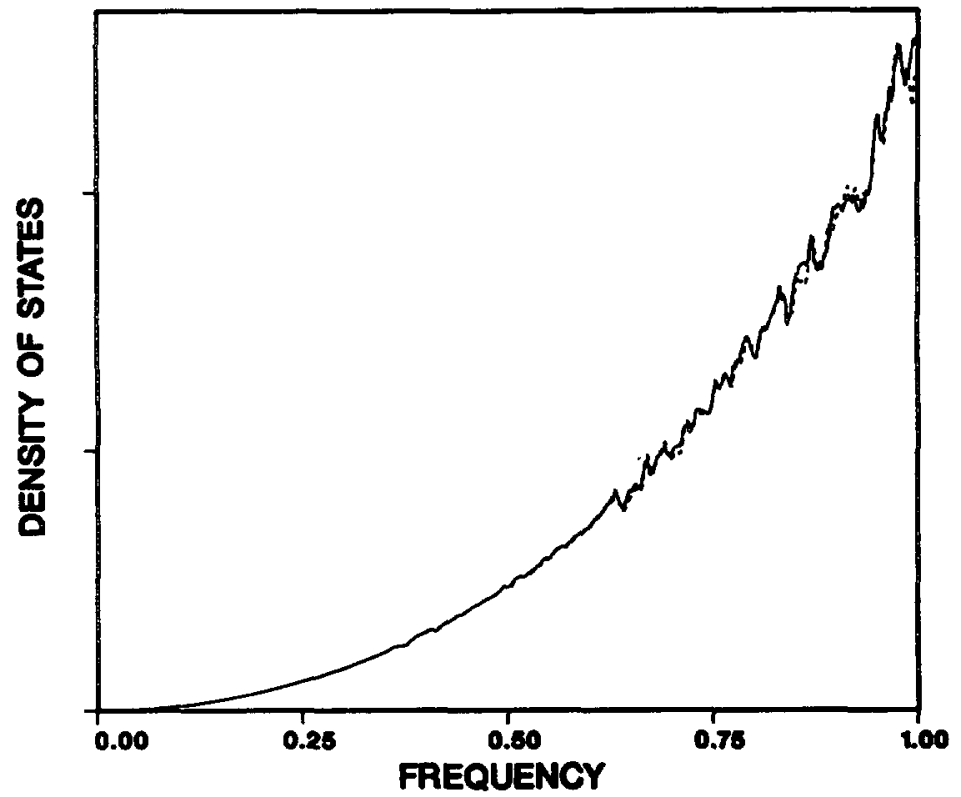

Fig. 8. - The normalized density of states at low frequency for two randomized 3/2-approximants (solid line and dashed line), obtained from quadratic grid 16 interpolation. 
interpolation. Both curves are almost identical, illustrating that characteristic deviations from the normal $\omega^{2}$-behaviour can very well be investigated by considering the contributions from only one octant, which we will do from now on.

In figure 5 the DOS is shown for the 3/2-approximant using a grid 16 quadratic interpolation (solid line), a grid 8 quadratic interpolation (dashed line) and a grid 16 linear interpolation (dotted line). The curves that result from the quadratic and linear grid 16 interpolations are almost identical, which is an indication that the DOS has converged at this grid density. Figure 6 shows the DOS for the 5/3-approximant using a grid 16 quadratic interpolation (solid line) and a grid 16 linear interpolation (dashed line). The dotted line in this picture represents the DOS for a bcc structure, and shows that the number of low frequency states is relatively higher for the i-PT approximants. Note that for the results of figures 4, 5 and 6 (and also for Figs. 7 and 8 presented later on) an appropriate normalization has been applied, and the scale on the $y$-axis is the same in each of these pictures. The higher density of low frequency states relative to the bcc result may be partially due to the fact that on average the atoms in the i-PT structure are connected to fewer neighbours $(\approx 13.2)$ than in the bcc structure model (14). However, this explanation is not complete because the same effect, although to a lesser extent, was also observed when a comparison was made with a fcc structure model, where each atom was connected to 12 neighbours [1]. Obviously the typical tiling order leads to a slightly higher density of low frequency modes.

Comparing the figures 4,5 and 6 we observe that the first significant deviation from the normal $\omega^{2}$-behaviour occurs at about $\omega=0.67$ in each of the three cases, although this value varies somewhat for the three approximants. In the range below this frequency the curve represents an almost smooth $\omega^{2}$-behaviour. In particular for the 3/2-and 5/3-approximants this frequency range is far beyond the range of the three acoustic branches, but obviously the gaps occurring in the dispersion in this part of the spectrum are so small that they do not give rise to large deviations in the DOS. Also for the incommensurate limit we do not expect large deviations from the $\omega^{2}$-behaviour in this frequency region, since the results for the three successive approximants do not support such an expectation. Clearly the widths of the gaps at the Brillouin zone boundaries at low frequencies do not increase for higher approximants, and no new large gaps do appear.

Since one of the three neighbour distances is considerably smaller than the other two it seems realistic to put a stronger spring between two atoms at this distance. We have again determined the DOS at low frequency for the 3/2-approximant, where now the spring constant for the short bond length was taken to be equal to 2 or 3 times the value of the spring constant for the other bond lengths. In both cases, the two different spring constants where chosen such that $\sum_{i=1}^{N} \sum_{,=1}^{N} \alpha_{i i^{\prime}}$ was equal to $14 N$, as is the former case. Here $\alpha_{1 \prime}$ is the spring constant between atom $i$ and $i^{\prime}$ and $N$ is the number of atoms in the unit cell. The result is shown in figure 7. Comparing it with the result of figure 5 we conclude that the characteristic features of the DOS at low frequency are hardly affected by putting stronger springs between the closer neighbours. The most important difference with the former result is that the proportionality constant of the global $\omega^{2}$-behaviour increases when the relative difference between the strengths of the springs is increased. This is due to the fact that, to compensate for the stronger spring between the closer neighbours, the spring constant for both other bond lengths becomes smaller. Since the latter bond lengths occur much more frequently than the short one, the value of the spring constant for these bond lengths is the most important for the long wavelength modes. This also explains the shift of the pseudogaps to lower frequencies, when the difference in the spring constants is increased.

We have also studied the effect of randomization. Figure 8 shows the DOS for two 
randomized 3/2-approximants using a quadratic grid 16 interpolation. We observe that the pseudogap at about $\omega=0.67$ has almost disappeared in both cases. Such an smoothening effect of randomization on the DOS was also observed in similar calculations on the phonon spectrum for an octogonal tiling model in two dimensions [16]. Obviously the very specific local order, which is present only in the perfect approximants, gives rise a to characteristic pseudogap at low frequency. For the randomized approximants the local order is partially destroyed.

\section{The dynamical structure factor.}

The dynamical response of a crystal in inelastic neutron scattering experiments is usually described by the dynamical structure factor $S(\mathbf{k}, \omega)$, which is proportional to the measured coherent cross section for scattering processes with momentum transfer $\hbar \mathbf{k}$ and energy transfer $\hbar \omega$. Within the harmonic approximation the dynamical structure factor for one phonon processes is given by:

$$
S(\mathbf{k}, \omega)=\sum_{\mathbf{k}} \sum_{j \mathbf{q}} \delta(\mathbf{k}+\mathbf{q}-\mathbf{K}) \frac{\left|H_{\mathbf{k}}^{\prime}\right|^{2}}{\omega_{j}(\mathbf{q})}\left[n_{j}(\mathbf{q}) \delta\left(\omega+\omega_{j}(\mathbf{q})\right)+\left(1+n_{j}(\mathbf{q})\right) \delta\left(\omega-\omega_{j}(\mathbf{q})\right)\right]
$$

where the summations over $\mathbf{K}$ and $\mathbf{q}$ are, respectively, summations over the reciprocal lattice vectors and all wave vectors inside the first Brillouin zone, $n_{j}(\mathbf{q})$ the Bose-Einstein occupation number for the $j$-th eigenstate at wavevector $q$ with frequency $\omega_{j}(q)$, and $H_{\mathbf{k}}^{j}$ is defined for each eigenstate $j$ by

$$
H_{\mathbf{k}}^{\prime}=\sum_{d} \frac{b_{d}}{M_{d}}\left(\mathbf{k} \cdot \mathbf{u}_{d}^{\prime}(\mathbf{q})\right) \mathrm{e}^{-W_{d}(\mathbf{k})+i \mathbf{k} \cdot \mathbf{d}}
$$

where the summation runs over all atoms in the unit cell. In (10), each atom $d$ is characterized by its mass $M_{d}$, its equilibrium position d, its coherent scattering length $b_{d}$, the Debye-Waller factor $\exp \left(-W_{d}(\mathbf{k})\right)$ and the displacement $\mathbf{w}_{d}^{j}(\mathbf{q})$ in the $j$-th eigenstate at wavevector $q$. For a derivation of these expressions, see for example reference [17].

The thermal factors $n_{j}(\mathbf{q})$ and $1+n_{j}(\mathbf{q})$ correspond to processes in which a phonon is absorbed and emitted, respectively. In the low temperature limit, $n_{j}(\mathbf{q})$ goes to zero and the term in which phonons are emitted is the dominant one. The Debye-Waller factor induces a reduction of the intensity, but at low temperatures the exponent $W_{d}(\mathbf{k})$ is small and the DebyeWaller factor becomes unimportant. In our models all coherent scattering lengths and masses are equal, and at low temperatures we obtain then, omitting also the factor $1 / \omega_{j}$, which is not relevant for a qualitative study of the dynamics, the following simplified expression for the dynamical response of the model :

$$
I(\mathbf{k}, \omega)=\sum_{j}\left|\sum_{d}\left(\mathbf{k} \cdot \mathbf{u}_{d}^{j}(\mathbf{q})\right) \mathrm{e}^{\mathbf{k} \cdot \mathbf{d}}\right|^{2} \delta\left(\omega-\omega_{j}(\mathbf{q})\right)
$$

where $\mathbf{q}$ is equal to the momentum transfer $\mathbf{k}$ modulo a reciprocal lattice vector $\mathbf{K}$.

In experiments, one normally starts measuring at wave vectors with strong Bragg intensity and one expects to see a pronounced dispersion curve close to strong Bragg peaks. For normal periodic structures, halfway between two neighbouring Bragg peaks is the Brillouin zone boundary. At this boundary one expects to see a bending of the dispersion curve and, depending on the decoration of the unit cell, possibly a gap. For a quasicrystal, halfway between two strong Bragg peaks is a pseudo-Brillouin zone boundary, and one may wonder whether the behaviour of the dynamical response when moving from the strong Bragg peak to the pseudo-Brillouin zone boundary is similar to that of a normal crystal. 
The elastic scattering response, giving the Bragg spots, is determined by the static structure factor $S_{\mathbf{K}}$. For our models $S_{\mathbf{K}}$ is given by :

$$
S_{\mathbf{K}}=\sum_{d} \mathrm{e}^{i \mathbf{K} \cdot \mathrm{d}},
$$

where the summation runs over all atoms in the unit cell of the i-PT approximant. The intensity of the Bragg peak at $\mathbf{K}$ is proportional to $\left|S_{K}\right|^{2}$. Figures $9 \mathrm{a}$ and $9 \mathrm{~b}$ show the Bragg spots, as
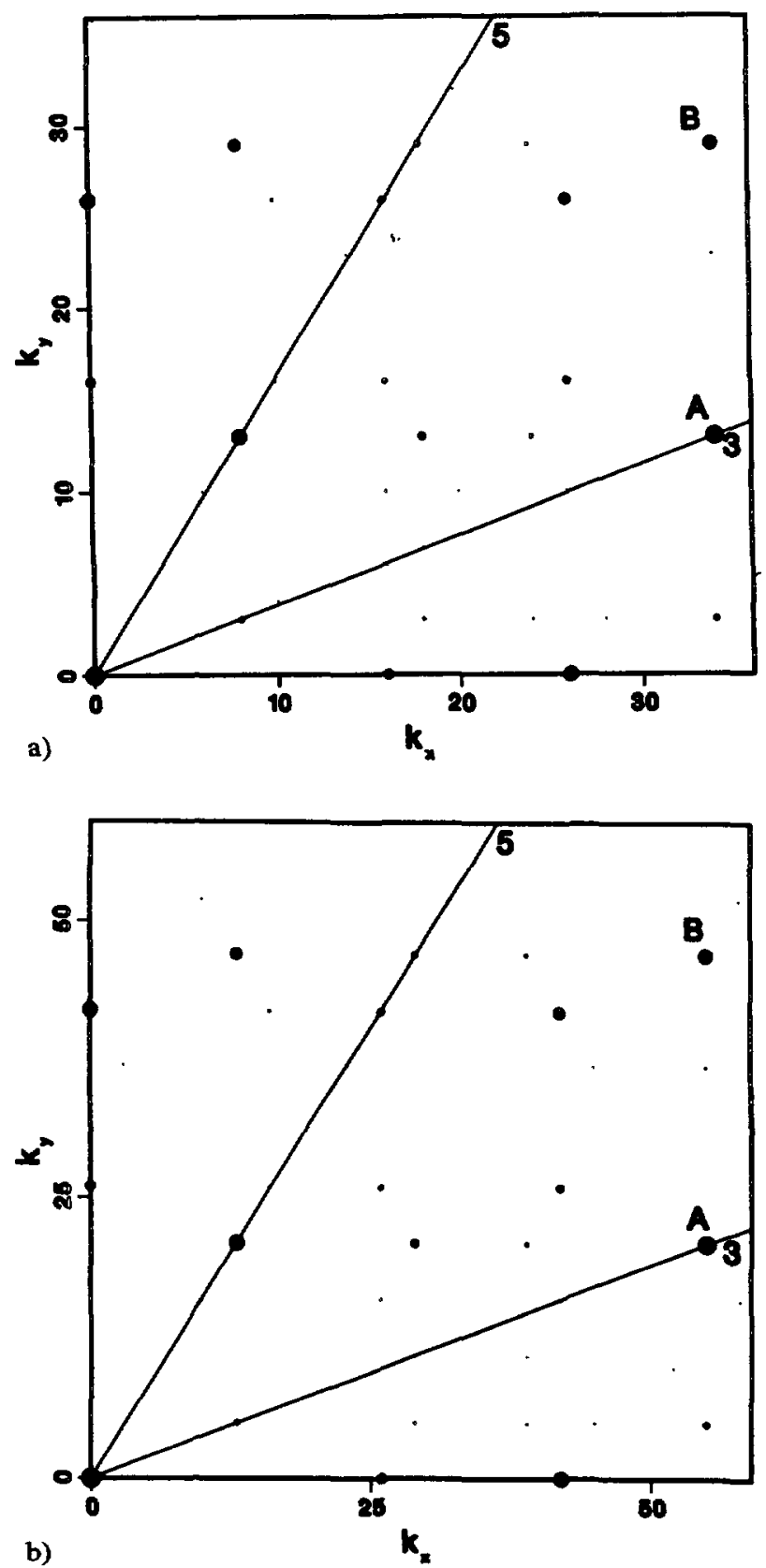

Fig. 9. - a) The intensity of the Bragg peaks in the $k_{z}=0$ plane for the symmetrized 3/2-approximant. The labels on both axis are in units of $2 \pi / a$. b) As in figure 9a, but for the symmetrized 5/3-approximant. 
calculated according to (12), in the $k_{z}=0$ plane for the symmetrized 3/2-approximant and 5/3approximants, respectively. These two pictures are very similar, which means that convergence takes place very rapidly for successive approximants. In these plots, the k-components $k_{x}$ and $k_{y}$ are given in units of $2 \pi / a$. The difference in scale (in these units) between the two plots is in agreement with the fact that the lattice constant $a$ grows with a factor $\phi$ between successive approximants. For example, spot $A$ has labels $(21,8),(34,13)$ and $(55,21)$ for the 2/1-, 3/2- and 5/3-approximants respectively, and these numbers are all members of the Fibonacci sequence $\left\{F_{n}\right\}$, defined by the recursion $F_{n+1}=F_{n}+F_{n-1}$ with $F_{-1}=F_{0}=1$. It holds that $\phi=\lim F_{n+1} / F_{n}$, which explains the scaling behaviour of the $\left(k_{x}, k_{y}\right)$-labels.

Notice also that figure 9 shows much similarity with data from $\mathrm{X}$-ray diffraction measurements of real i-phase quasicrystals, in spite of the simplicity of our model. This is a consequence of the common higher dimensional lattice symmetry, which exists both for our model and for a realistic structure. The strong Bragg peaks correspond to $F$-points in the higher dimensional reciprocal space which are relatively close to the physical space.

To get a first impression of the dynamical response of our models, we have determined the dynamical response $I(\mathbf{q}, \omega)$ along the path $\mathrm{AB}$ for symmetrized approximants, taking into account only those wave vectors yielding a real dynamical matrix. These are the reciprocal lattice points and the points in the middle between two lattice points. For these $k$ points the dynamical matrix has respectively $T_{h}$ and $D_{2 h}$ symmetry, which we used to reduce the dynamical matrix to block form. In view of the computation time and storage capacity that is needed, this reduction is very useful. The delta peaks of the expression (11) can be weakened by using :

$$
\delta\left(\omega-\omega_{1}\right)=-\frac{1}{\pi} \operatorname{Im} \frac{1}{\omega+i \varepsilon-\omega_{1}}
$$

with $\varepsilon$ a small positive number, which governs the width of the peaks. Such a broadening is also present in experimental data, due to anharmonic effects and the finite resolution of the experimental devices.

Figure 10a shows the dynamical response $I(\mathbf{q}, \omega)$ for the symmetrized 3/2-approximant when moving from $A$ to $B$, and figure $10 \mathrm{~b}$ shows the same for the symmetrized $5 / 3$ approximant when moving in the opposite direction, from B to $A$. In these calculations we have taken $\varepsilon$ equal to 0.05 . Details that may disappear by using this finite value for $\varepsilon$ are not expected to be seen in experiments. Recall that the total frequency support for our models is about 3.2. Both pictures roughly show a linear dispersion up to rather high frequencies. In fact, we can distinguish two linear dispersions starting from the strong Bragg positions, one corresponding to transversal and the other one to longitudinal polarization of the modes relative to the $k_{y}$-direction. When approaching the pseudo-Brillouin zone boundary, bending of the dispersion curves is observed. In figure 10a the response due to longitudinal polarization is much weaker than the response due to transversal polarization, in agreement with the fact that the projection of longitudinal displacements on $\mathbf{k}$ close to $\mathrm{A}$ is smaller than that of transversal displacements. For k-vectors close to $\mathrm{B}$ the intensity for the longitudinal modes is much bigger, which is clearly shown in figure $10 \mathrm{~b}$. For comparison, figure $10 \mathrm{c}$ shows the result for a bcc structure, moving between two $\Gamma$-points, from $(5,5,0)$ to $(5,3,0)$ in units of $2 \pi / c$, where $c$ is the edge length of the smallest cubic unit cell for a bcc structure, containing two atoms per unit cell, so that according to (3) $c=2^{1 / 3}$ This path is comparable to the path BA for the i-PT approximant. Note that the point $(5,4,0)$ is not a $\Gamma$-point of the reciprocal lattice for the bcc-structure, due to the fact that the cubic unit cell is not a primitive unit cell. From figures $10 \mathrm{a}-10 \mathrm{c}$ it may be concluded that the results for the i-PT approximants are globally similar to those of a normal periodic structure. 


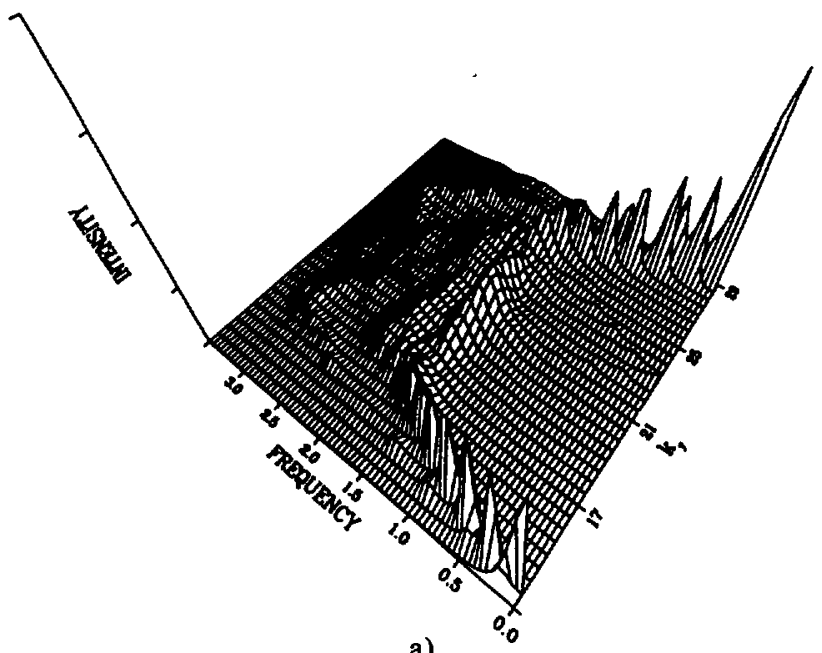

a)

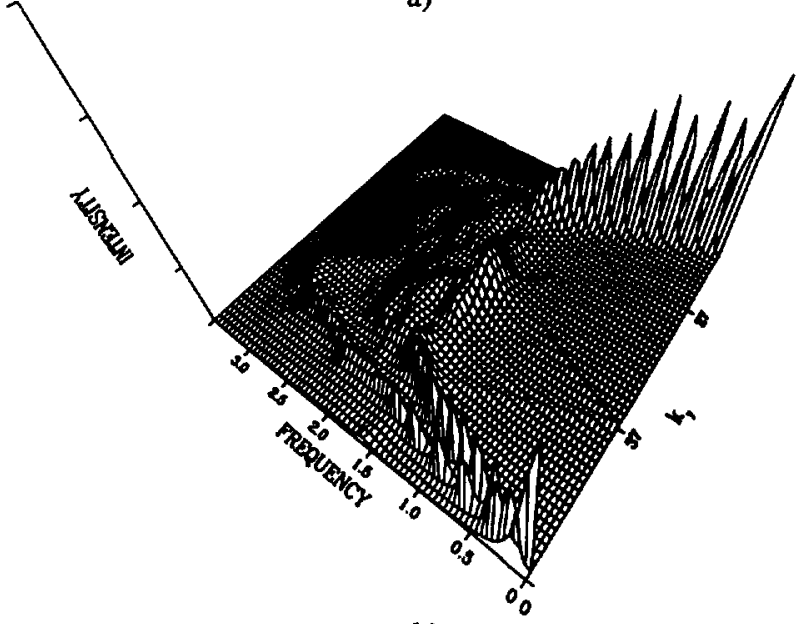

b)

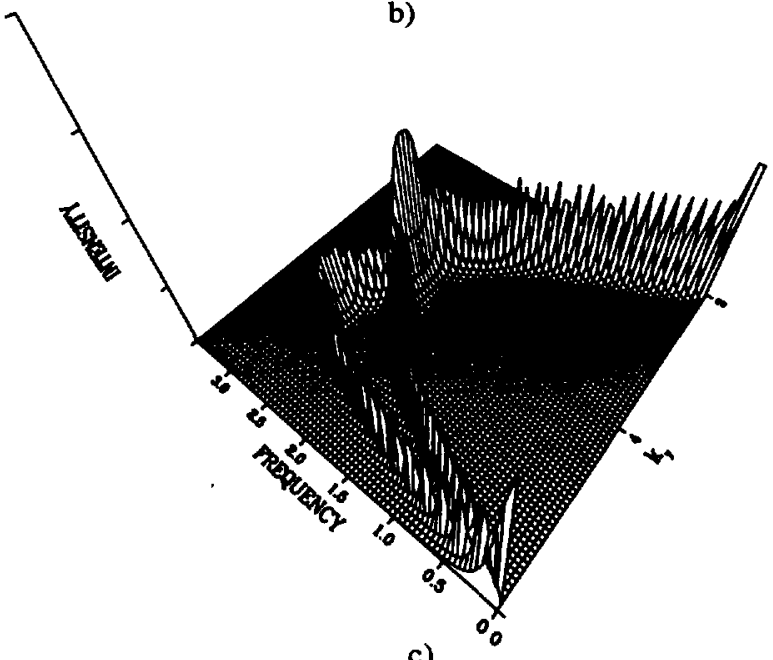

c)

Fig. 10. - a) The dynamical response along the path from A to B (see Fig. 9a) for the symmetrized 3/2approximant. b) The dynamical response for the symmetrized 5/3-approximant, along the path from $B$ to A (see Fig. 9b). c) The dynamical response for a bcc model along a similar path as in figure 10b. 
The dynamical response due to modes with a specific polarization can be represented by the following function :

$$
I_{\dot{\mathrm{e}}_{\mathrm{p}}}(\mathbf{k}, \omega)=\frac{1}{N} \sum_{j}\left|\sum_{d}\left(\hat{\mathbf{e}}_{\mathrm{p}} \cdot \mathbf{u}_{d}^{\prime}(\mathbf{q})\right) \mathrm{e}^{\prime \mathbf{k} \cdot \mathrm{d}}\right|^{2} \delta\left(\omega-\omega_{j}(\mathbf{q})\right)
$$

where $\hat{\mathbf{e}}_{\mathrm{p}}$ a unit polarisation vector. This function can be regarded as the imaginary part of a diagonal matrix element of the resolvent operator:

$$
I_{\dot{\mathbf{e}}_{\mathrm{p}}}(\mathbf{k}, \omega)=-\frac{2 \omega}{\pi} \lim _{\varepsilon \rightarrow 0} \operatorname{Im}\left\langle\mathbf{u}_{\dot{e}_{\mathrm{p}}}^{(\mathbf{k})}\left|\left[(\omega+i \varepsilon)^{2}-H^{(\mathbf{k})}\right]^{-1}\right| \mathbf{u}_{\dot{e}_{\mathrm{p}}}^{(\mathbf{k})}\right\rangle
$$

where $\mathbf{u}_{\hat{e}_{\mathrm{p}}}^{(\mathbf{k})}$ is the $3 N$ components vector with components $N^{-1 / 2} \hat{\mathbf{e}}_{\mathrm{p}} \exp (i \mathbf{k} \cdot \mathbf{d})$ for the atom at position $\mathbf{d}$, and $H^{(\mathbf{k})}$ is the dynamical matrix at wave vector $\mathbf{k}$. The function $I_{\dot{e}_{\mathrm{p}}}(\mathbf{k}, \omega)$ can be calculated using a recursion method [9]. In this method, $H^{(\mathbf{k})}$ is tridiagonalized recursively by the Lanczos algorithm described in Appendix A, taking as starting vector the vector $\mathbf{u}_{e_{p}}^{(k)}$ The coefficients of the tridiagonal matrix are directly related to the coefficients of a continued fraction expansion of the resolvent operator. The termination of the continued fraction expansion is a problem which has been the subject of several papers [18, 19]. Another problem is that the tridiagonalization process becomes numerically unstable after some finite number of steps (see Appendix A). Therefore it is believed that one can only obtain results with limited accuracy. In the following we propose an alternative method to obtain the relevant information from the truncated tridiagonalization, and show that our method gives quite accurate results, in spite of the numerical deficiencies of the tridiagonalization process.

Assuming that the tridiagonalization algorithm is exact, we can write the truncated process in matrix form as :

$$
T_{m}=V_{m}^{\dagger} H^{(\mathbf{h})} V_{m}
$$

where we have truncated after the $m$-th step. $T_{m}$ is a tridiagonal matrix of order $m$ and $V_{m}$ is the $m \times n$ transformation matrix whose first column is the vector $\mathbf{u}_{\mathbf{e}_{\mathrm{p}}}^{(\mathbf{k})}$ Substitution in (15) gives :

$$
I_{\dot{\mathbf{e}}_{\mathrm{p}}}(\mathbf{k}, \omega)=-\frac{2 \omega}{\pi} \lim _{\boldsymbol{r} \rightarrow 0} \operatorname{Im}\left\langle\mathbf{v}_{1}\left|(\omega+i \varepsilon)^{2}-T_{m}^{-1}\right| \mathbf{v}_{1}\right\rangle
$$

where the $m$ components vector $\mathbf{v}_{1}$ is a unit vector whose first component is equal to 1 , whereas all other components are equal to 0 . Using a matrix inversion formula we obtain :

$$
I_{\dot{\mathbf{e}}_{\mathbf{p}}}(\mathbf{k}, \omega)=-\frac{2 \omega}{\pi} \lim _{\varepsilon \rightarrow 0} \operatorname{Im} \frac{\operatorname{det}\left[(\omega+i \varepsilon)^{2}-\Delta_{11}\right]}{\operatorname{det}\left[(\omega+i \varepsilon)^{2}-T_{m}\right]}
$$

where $\Delta_{11}$ is the cofactor of the first diagonal element of $T_{m}^{(\mathbf{k})}$. If $\lambda,(j=1, \ldots, m)$ are the eigenvalues of $T_{m}^{(\mathbf{k})}$ and $\mu_{j}(j=1, \ldots, m-1)$ the eigenvalues of $\Delta_{11}$ we obtain:

$$
I_{\dot{\mathbf{e}}_{\mathbf{p}}}(\mathbf{k}, \omega)=-\frac{2 \omega}{\pi} \operatorname{Im} \frac{\prod_{j=1}^{m-1}\left(\omega^{2}-\mu_{j}+2 i \varepsilon \omega\right)}{\prod_{j=1}^{m}\left(\omega^{2}-\lambda_{j}+2 i \varepsilon \omega\right)}
$$


where $\varepsilon$ is some small positive number and the term $\varepsilon^{2}$ has been neglected. The eigenvalues of the tridiagonal matrices can be found very efficiently by the QL algorithm, and from these the imaginary part of $I_{\dot{e}_{p}}(k, \omega)$ can be computed without numerical difficulties. Since the tridiagonalization process is numerically unstable, formula (16) is not exactly true any more after a certain number of recursion steps. However, on the basis of the fact that the recursion is locally stable, we can still use the same expression and see what the results look like. By doing this we observed that convergence is obtained in the sense that for fixed $k$ the function $I_{\dot{e}_{p}}(\mathbf{k}, \omega)$ does not change any more under enlargement of the tridiagonal matrix. This does not automatically imply that the converged result is the correct result. To investigate this we have compared the result with a more reliable result, which can be obtained by computing all eigenvalues $\omega_{J}^{2}$ and eigenvectors $\left|\alpha_{J}\right\rangle$ of $H^{(\mathrm{k})}$ and then evaluating :

$$
I_{\dot{e}_{p}}(\mathbf{k}, \omega)=-\frac{2 \omega}{\pi} \operatorname{Im} \sum_{j=1}^{3 N} \frac{\left|\left\langle\mathbf{u}_{\mathbf{e}_{p}}^{(\mathbf{k})} \mid \alpha_{j}\right\rangle\right|^{2}}{\left(\omega^{2}-\omega_{j}^{2}+2 i \varepsilon \omega\right)}
$$

In figure 11 the function $I_{\dot{e}_{\mathrm{p}}}(\mathbf{k}, \omega)$ at $\mathbf{k}=4 \pi / \alpha$ is shown for the symmetrized $3 / 2$ approximant. In this picture, the results for $\varepsilon$ equal to 0.05 and $\varepsilon$ equal to 0.01 are compared, obtained by using the recursion formula (19). Clearly, the latter result contains many more details. However, the price to be paid for these details is that more recursion steps are required to obtain convergence, about 300 . For $\varepsilon$ equal to 0.05 the number of recursion steps required to obtain convergence was only 75 . Note that with convergence we mean convergence for each $\omega$ in the interval $[0,3,5]$. In practice one can obtain reasonable results with fewer recursion steps. The dotted line in figure 11, which represents the result obtained by explicit diagonalization of $H^{(\mathbf{k})}$ and using (20) with $\varepsilon$ equal to 0.01 , demonstrates that our approach to the recursion method gives quite good results.

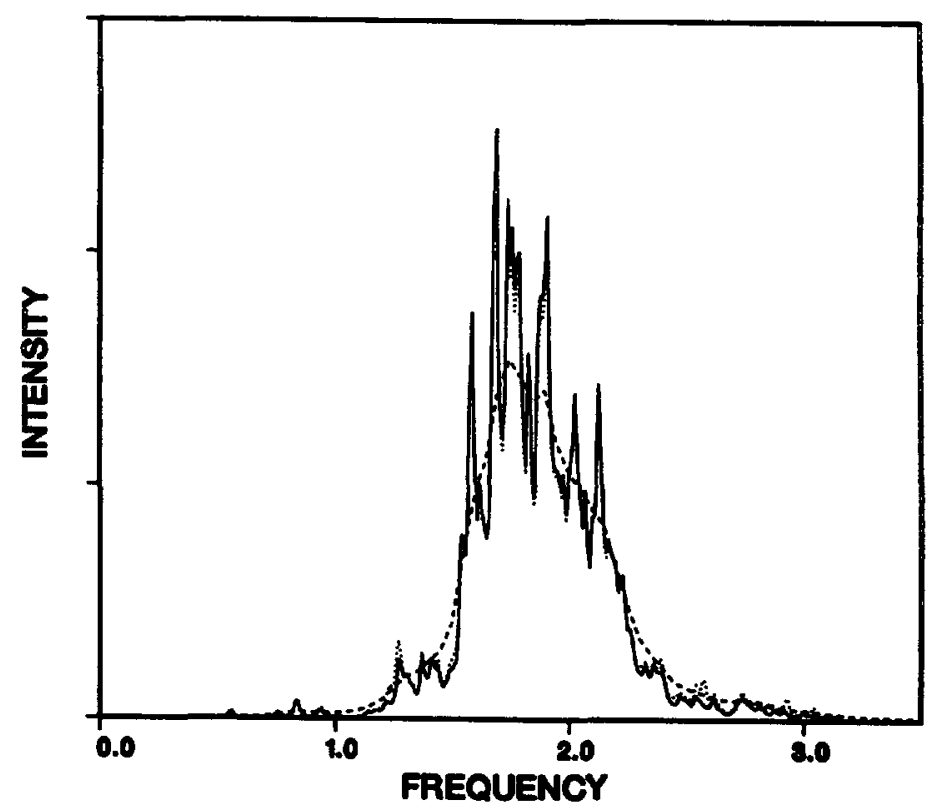

Fig. 11. - The dynamical response at wave vector $k=4 \pi / a$ for the symmetrized 3/2-approximant, using the recursion method with $\varepsilon=0.01$ (solid line) and $\varepsilon=0.05$ (dashed line). The dotted line is the result obtained by explicit diagonalization and using expression (20) with $\varepsilon=0.01$. 
The fact that convergence is obtained using the recursion method may be understood on the basis of what is explained in apppendix $A$. There it is stated that the eigenvalues, which occur in the spectrum of $T_{m}$ as a consequence of the instability of the recursion process, will also occur as eigenvalues of the matrix $\Delta_{11}$, which means that the corresponding factors occurring in (19) cancel.

The dynamical response for the 8/5-approximant at wave vectors along the $k_{\mathrm{A}}$-direction, obtained using the recursion method with $\varepsilon=0.05$, is shown in figures $12 \mathrm{a}$ and $12 \mathrm{~b}$ for transversal and longitudinal polarization, respectively. These pictures clearly show a strong response along a linear dispersion curve up to rather high frequencies. The isotropy of the dispersion is demonstrated by figure $12 \mathrm{c}$, where the response from longitudinal modes along the quasi-five-fold direction $(1, \phi, 0)$ is shown.

The behaviour of the dynamical response for the quasiperiodic structure may be obtained by studying the convergence of the results for a number of successive approximants. Figure 13 shows the maximal intensity due to transversal polarization at wave vectors along the quasifive-fold direction $(1, \phi, 0)$ for the $2 / 1-, 3 / 2-, 8 / 5$-approximants and a bcc structure. The labels on the $k_{x}$-axis are in units of $2 \pi / c$, where $c$ is related to the bcc lattice as defined above. The value of $\varepsilon$ was taken equal to 0.05 . The picture shows that global convergence is obtained already for the lowest approximants. The maximal possible intensity is the intensity at $\mathbf{k}=0$. Along the dispersion, the intensity has decreased to half the maximal intensity at about $k=4 \pi / 5 c$, corresponding to a frequency of about 1.4, which is more than one third of the total frequency range. For the bcc lattice the intensity stays close to the maximal intensity along almost the entire dispersion, in spite of the fact that the $(1, \tau, 0)$ direction is not a lattice direction. For longitudinal modes the frequency at which the intensity has decreased to half the maximal intensity is about $\omega=1.75$.

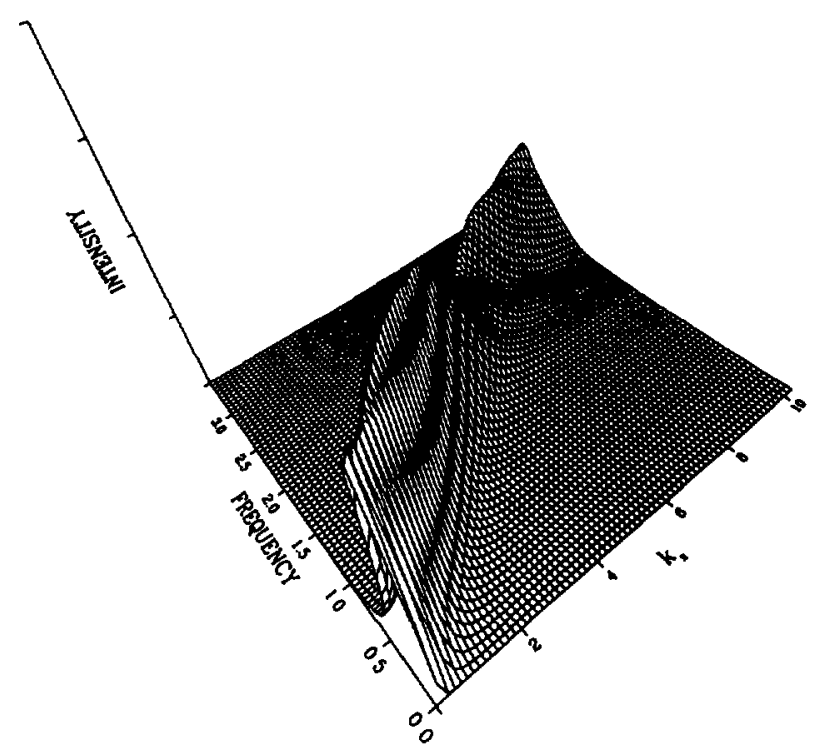

a)

Fig. 12. - a) The dynamical response $I_{\hat{e}_{\mathrm{p}}}(\mathbf{k}, \omega)$ for the 8/5-approximant for longitudinal polarization, at wave vector along the $k_{1}$-direction. 


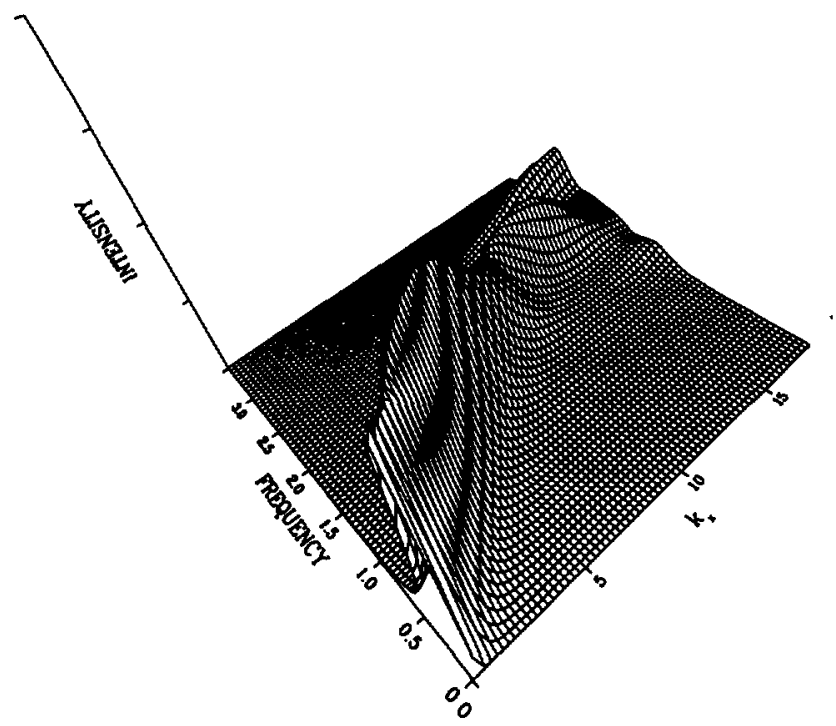

b)

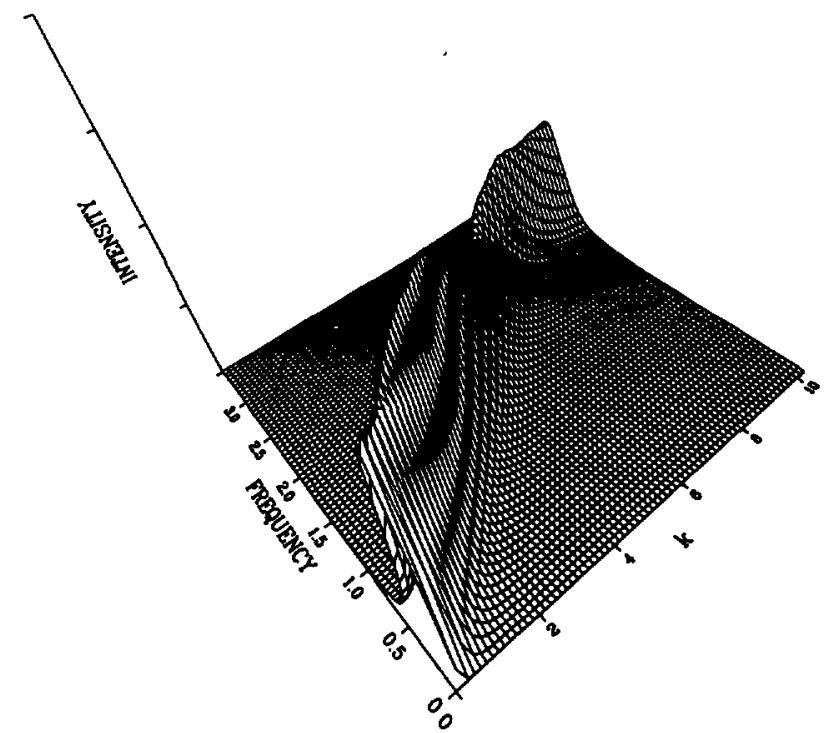

c)

Fig. 12. - b) The dynamical response for the 8/5-approximant for transversal polarization, at wave vector along the $k_{1}$-direction. c) The dynamical response for the $8 / 5$-approximant for longitudinal polarization, at wave vector along a quasi-five-fold direction.

The convergence of the fine structure of $I_{\dot{e}_{p}}(\omega, \mathbf{k})$, when tending to the quasiperiodic limit, can be studied by taking a smaller value for $\varepsilon$. We have considered the response from transversal polarization at some fixed wave vectors along the quasi-five-fold direction with $\varepsilon$ equal to 0.01 . Figure $14 \mathrm{a}$ shows the results for the wave vectors $\mathbf{k}=2 \pi / 5 c$ and $4 \pi / 5 c$ in one picture, for the $2 / 1-, 5 / 3-$ and $8 / 5$-approximants. Strong intensity with little structure is observed at these wave vectors. Obviously the optical branches which appear at 


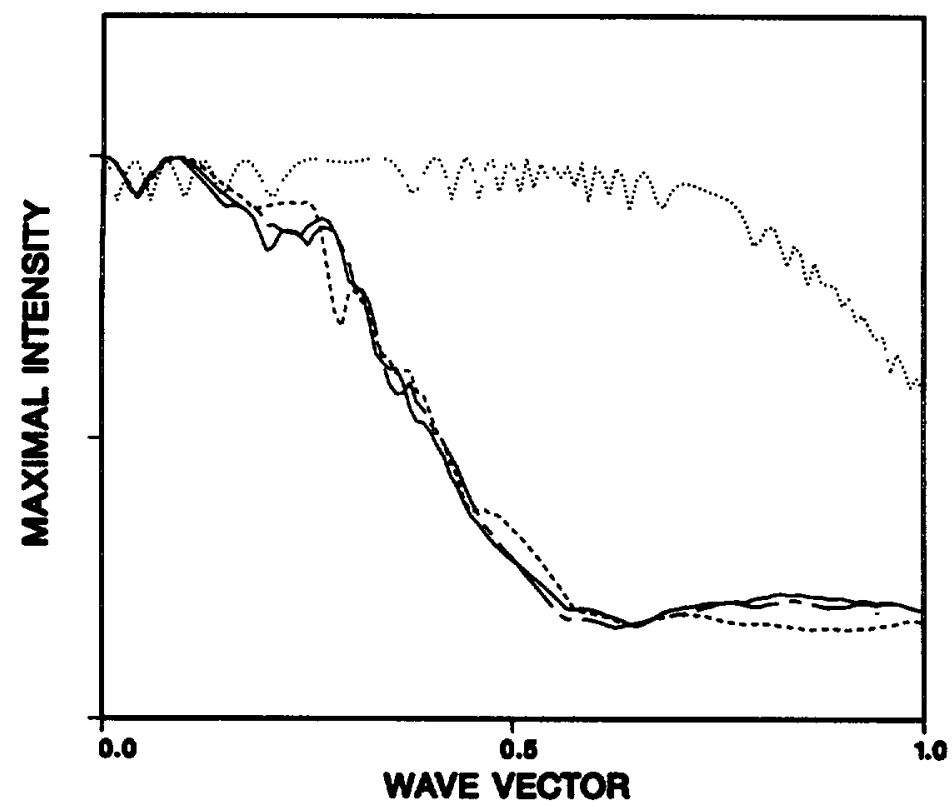

Fig. 13. - The maximal response for the 2/1-, 3/2- and 8/5-approximant (respectively solid, chaindashed and dashed line) and a bcc structure (dotted line) for transversal polarisation, with wave vector along the quasi-five-fold direction, as function of the wave vector in units of $2 \pi / c$ with $c$ corresponding to the bcc lattice.

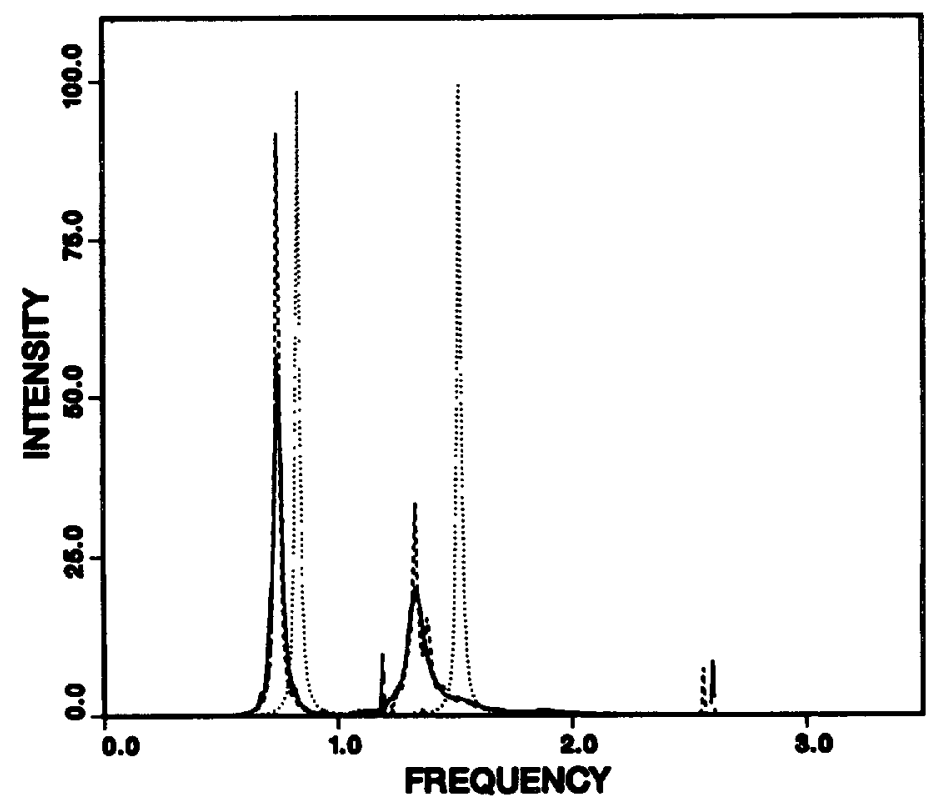

Fig. 14. - a) The dynamical response for the 2/1-, 5/3- and 8/5-approximant (respectively solid, chaindashed and dashed line) and a bcc structure (dotted line) due to transversal polarization, with wave vector along the quasi-five-direction, at $k$ equal to $2 \pi / 5 c$ (peaks at $\omega \approx 0.8$ ) and at $4 \pi / 5 c$ (peaks at $\omega \approx 1.3$ ). 


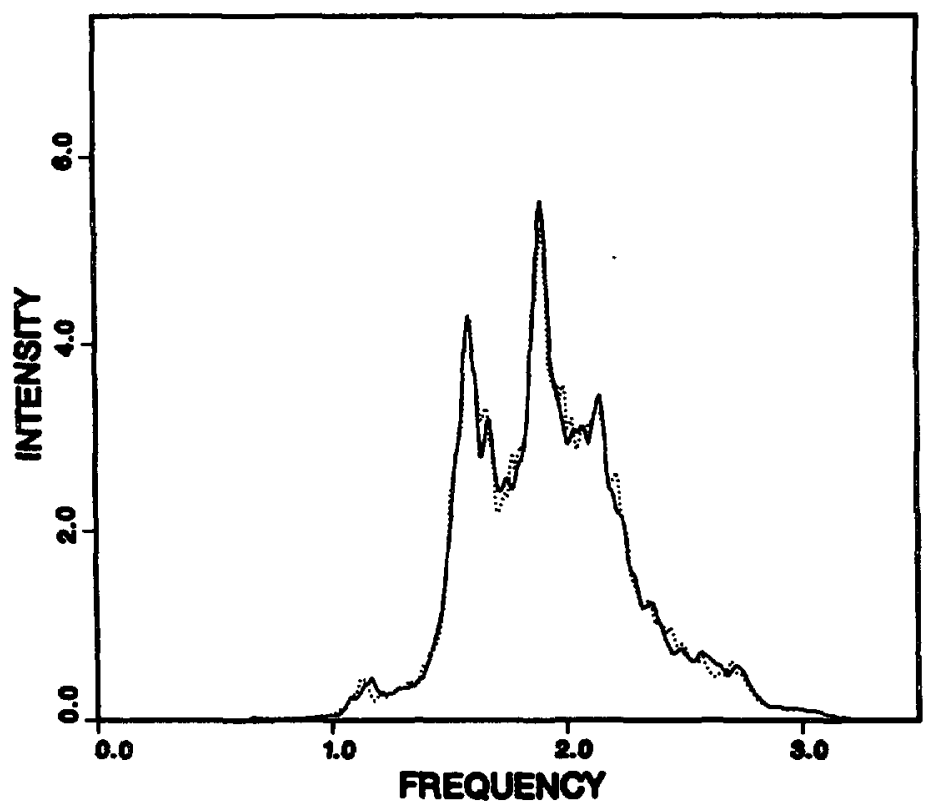

b)

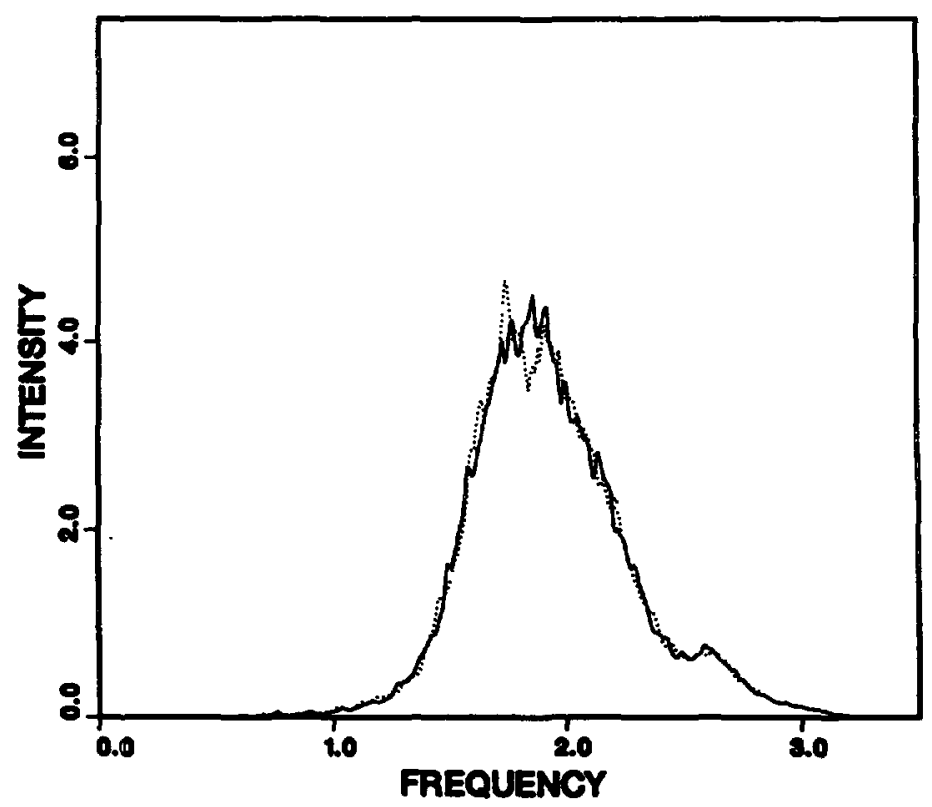

c)

Fig. 14. - b) As in figure 14a, but at $k=6 \pi / 5 c$, for the 5/3- and 8/5-approximants (respectively solid and dotted line). c) As in figure 14a, but for two randomized 5/3-approximants (respectively solid and dotted line), at $k=6 \pi / 5 c$. 
arbitrary low frequencies (see Sect. 2) hardly contribute to the dynamical response at low frequencies. The intensities for the $2 / 1$-approximants are stronger, but the results for both larger approximants seem to indicate that convergence has been obtained. Figure $14 \mathrm{~b}$ shows the result at $k=6 \pi / 5 c$ for the 5/3- and 8/5-approximants, and illustrates that at higher frequencies the peak is broadened, and much of its intensity is lost. Moreover, much more structure can be seen. Nevertheless, convergence is obtained also at such high frequencies, when tending to higher approximants, which is remarkable. This means that also in calculations of the dynamical structure factor for more realistic models of quasicrystals, the commensurate approximation may be expected to be a good approximation for the entire frequency range. Finally, figure $14 \mathrm{c}$ presents the result at the same wave vector for two randomized 5/3-approximants, showing that some smoothening is induced by randomization. This is in agreement with the results for the total DOS [1], and is obviously a consequence of the loss of order induced by randomization.

\section{Concluding remarks.}

Although our models are far from being realistic, we still believe that qualitatively our results are very well suited to gain insight into the phonon properties of quasicrystals at low frequencies.

There is an evident scaling behaviour of the lowest phonon branches for successive approximants, which makes the behaviour of the low frequency spectrum in the quasiperiodic limit predictable. Since the widths of the gaps relative to the bandwidths vanish in the low frequency limit, the density of states starts normal quadratically at $\omega=0$. However, already at relatively low frequencies, deviation from the $\omega^{2}$ behaviour can be expected for the perfect tilings in the form of pseudo gaps. For randomized tilings these pseudogaps are much smaller.

One can expect to measure, by inelastic neutron scattering measurements, a strong dynamical response along a normal linear dispersion up to rather high frequencies, and bending of the dispersion occurs near the pseudo-Brillouin zone boundaries. Moreover, the dispersion is isotropic, and the two transversal sound velocities converge to each other when tending to the quasiperiodic limit. At low frequencies, the dynamical structure factor shows a sharply peaked response with little structure along the dispersion curve. At higher frequencies, more structure and loss of intensity may be observed, in particular for perfect tilings. Also here, randomization has a smoothening effect. It appears that the use commensurate approximants is a good approximation for studying the dynamical properties of a quasicrystal, even to obtain insight into the fine structure.

\section{Appendix A.}

\section{Lanczos algorithm with no reorthogonalization.}

The Lanczos algorithm is a process in which a symmetric tridiagonal matrix $T$ is formed, which in exact arithmetic is, up to multiplicities, spectrally equivalent to a hermitian matrix $H$. During the process $H$ is not changed, and only matrix-vector multiplications are needed. Therefore, this algorithm is especially useful when $H$ is sparse. There are different variants of the basic Lanczos algorithm, which in exact arithmetic are equivalent. There have been [10] experiments with these variants, and the most stable version appears to be the following.

To start the process one needs a random unit vector $\mathbf{v}_{1}$, which is the first Lanczos vector, and which is supposed to contain a not very small projection on each of the orthogonal eigenspaces of $H$. Here and in the following we use « very small » to indicate a quantity of the order of the 
machine accuracy. Defining $\beta_{1}=0$ and $\mathrm{v}_{0}=0$, then for $j \geqslant 1$ the process is given by :

$$
\begin{aligned}
\mathbf{u}_{J} & =H \mathbf{v}_{J}-\beta_{J} \mathbf{v}_{J-1} \\
\alpha_{J} & =\mathbf{v}_{J}^{\dagger} \mathbf{u}_{J} \\
\mathbf{w}_{J} & =\mathbf{u}_{J}-\alpha_{J} \mathbf{v}_{J} \\
\beta_{J+1} & =\left(\mathbf{w}_{J}^{\dagger} \mathbf{w}_{J}\right)^{1 / 2} \\
\mathbf{v}_{J+1} & =\mathbf{w}_{J} / \beta_{J+1}
\end{aligned}
$$

where the numbers $\alpha_{1}(i=1,2 \ldots)$ and $\beta,(i=2,3 .$.$) are respectively the diagonal and$ subdiagonal (or superdiagonal) elements of the real symmetric tridiagonal matrix $T$, the socalled Lanczos matrix.

It is easy to show that in exact arithmetic the vectors $v_{J}(j=1, m)$ form an orthonormal basis for the space $\mathrm{K}_{m}=\operatorname{span}\left[\mathbf{v}_{1}, H \mathbf{v}_{1}, \ldots, H^{m-1} \mathbf{v}_{1}\right]$, which is known as the $m$-th Krylov subspace. Let $V_{m}$ be the $n \times m$-matrix whose $m$ columns are the vectors $\mathbf{v}_{j}(j=1, \ldots, m)$, where $n$ is the order of the matrix $H$. Then in matrix form (A.1) becomes:

$$
H V_{m}=V_{m} T_{m}+\beta_{m+1} \mathbf{v}_{m+1} \mathbf{e}_{m}^{\dagger}
$$

where $\mathbf{e}_{m}$ is a unit vector whose $m$-th component is equal to 1 , whereas all other components are equal to 0 . The matrix $T_{m}$ is said to be the orthogonal projection of $H$ onto $\mathrm{K}_{m}$.

For any finite matrix $H$ the process will stop whenever $\beta_{m+1}$ appears to be zero for some $m$. In exact arithmetic this will generally happen only when the order of $T_{m}$ is equal to the number of distinct eigenvalues of the matrix $H$. However, in practice the process does not stop, and $\beta$, will not even become small. This is due to the finite precision of the computer arithmetic, which leads to a considerable loss of orthogonality of the Lanczos vectors as soon as one or more eigenvalues of $H$ start to occur as eigenvalues of the matrix $T_{m}$ for some $m$ [11]. Nevertheless, the eigenvalues will converge in the sense that they occur in the spectrum for $T_{m}$ for all $m$ bigger than a certain fixed value. This is due to the fact that locally the recursion is stable, i.e. each new Lanczos vector is orthogonal to its immediate predecessors. The consequence of the fact that globally orthogonality is lost, is that the eigenvalues start to converge again, so that finally the same eigenvalue will be found twice or even more often among the eigenvalues of the matrix $T_{M}$ for large enough $M$.

A remedy for the loss of orthogonality is to reorthogonalize every new Lanczos vector with all its predecessors but this is of course a very expensive process, both for storage as well as for computation time reasons. Therefore, in what follows we focus on Lanczos procedures without reorthogonalization.

If $\mu$ is an eigenvalue of $T_{m}$ with corresponding normalized eigenvector $\mathbf{u}$, then the so-called Ritz vector $\mathbf{y}=V_{m} \mathbf{u}$ can be considered as an approximation of an eigenvector of $H$, and $(\mu, \mathbf{y})$ is denoted as a Ritz pair. There are $m$ such pairs for $T_{m}$. A measure for how good a Ritz pair approximates an eigenvalue/eigenvector pair of $H$ is given by the residual norm $|H y-\mu y|$ and in exact arithmetic this can be computed without explicit computation of the Ritz vector $\mathbf{y}$ by using (A2) :

$$
\begin{aligned}
|H \mathbf{y}-\mu \mathbf{y}| & =\left|H V_{m} \mathbf{u}-\mu V_{m} \mathbf{u}\right|=\left|\left(H V_{m}-V_{m} T\right) \mathbf{u}\right| \\
& =\left|\left(\beta_{m+1} \mathbf{v}_{m+1} \mathbf{e}_{m}^{+}\right) \mathbf{u}\right| \\
& =\beta_{m+1}|\mathbf{u}(m)|
\end{aligned}
$$

where $\mathbf{u}(m)$ denotes the $m$-th component of $\mathbf{u}$ and it is used that $\left|\mathbf{v}_{m+1}\right|=1$. This implies that there will be an eigenvalue $\lambda$ of $H$ such that :

$$
|\lambda-\mu| \leqslant \beta_{m+1}|\mathbf{u}(m)| \text {. }
$$


The expressions (A3) and (A4) show that whenever the last component $\mathbf{u}(m)$ of some eigenvector of $T_{m}$ is very small, then the corresponding $\operatorname{Ritz}$ pair $(\mu, y)$ is a good approximation for an eigenvalue-eigenvector pair of $H$. Due to the local stability of the recursion (A1) it has been shown that this result is still approximately true in the case of finite precision [11].

As a complement to these results Cullum and Willoughby make the following important statement [12].

\section{THE LANCZOS PHENOMENON.}

For large enough $M$ every distinct eigenvalue of $H$ will appear as an eigenvalue of the Lanczos matrix $T_{m}$ for all $m \geqslant M$.

Although they have not been able to prove this statement. they provide strong arguments and numerical evidence that it is true, by stressing the equivalence of the Lanczos tridiagonalization to the method of conjugate gradients for solving the linear system of equations $H \mathbf{x}=\mathbf{b}$ for positive definite $H$ and some $\mathbf{b}$, for which it is known that it converges, in spite of numerical instabilities.

Since the order $M$ of the tridiagonal matrix needed to achieve convergence for every distinct eigenvalue of $H$ might be much larger than the number of these distinct eigenvalues $k$ of $H$, one is faced with the problem of deciding which of the $M$ eigenvalues of $T_{M}$ are the eigenvalues of $H$. All other eigenvalues of $T_{m}$ are called " spurious ». Either these « spurious " eigenvalues are copies of the "good " eigenvalues, or they are eigenvalues which disappear when the size of the Lanczos matrix is increased. An eigenvalue of $T_{M}$ with a multiplicity bigger than one is considered as a « good $»$ eigenvalue. Such an eigenvalue just has converged more than once at this size of the Lanczos matrix. There are in principle two methods to decide which of the simple eigenvalues of $T_{M}$ are " good " ones. One method, which was described for example by Parlett and Reid [13], is based on the convergence of the eigenvalues of $T_{m}$ for increasing $m$. In this method an eigenvalue of $T_{m}$ is accepted as a «good » eigenvalue when it is also an eigenvalue of $T_{m+1}$ and of any $T_{m+k}$ with $k>1$. The other method, which was introduced by Cullum and Willoughby [12], is different in the sense that, instead of selecting the « good» eigenvalues, one identifies the «spurious» eigenvalues. Let $\dot{T}_{k}^{(m)}$ be the $(m-k+1) \times$ $(m-k+1)$ tridiagonal matrix obtained from $T_{m}$ by deleting the first $k-1$ rows and columns from $T_{m}$ (with $\hat{T}_{l}^{(m)}=T_{m}$ ). Then a simple eigenvalue of $T_{m}$ is considered « spurious » when it is also an eigenvalue of $\hat{T}_{2}^{(m)}$ In a careful analysis Cullum and Willoughby show why this procedure, which they call identification test, should work. Although it is not at all trivial to prove, we think it is intuitively clear : if a Ritz pair has converged for a certain size $m$ of the Lanczos matrix, the first component of the corresponding eigenvector $\mathbf{u}$ of $T_{m}$ will not be very small, since we assumed that the first Lanczos vector, which is the random starting vector, contains a not very small projection on each of the eigenspaces of $H$. But only if the same eigenvalue/vector pair starts to converge again, then the first component of the corresponding eigenvector $\mathbf{u}^{\prime}$ will be very small, so that the eigenvalue will also appear as an eigenvalue of $\hat{T}_{2}^{(m)}$. These eigenvalues will be identified as « spurious». In fact, they are either converged duplicates or eigenvalues which have not yet converged as duplicates of eigenvalues that have already converged.

The selection method of Cullum and Willoughby has an important advantage relative to the one of Parlett and Reid. After having eliminated the «spurious » eigenvalues, the remaining eigenvalues are considered as « good» approximations of eigenvalues of $H$, which might or might not have converged for some $\mathrm{m}$. So even before complete convergence has been achieved, these eigenvalues provide an early estimate of the entire spectrum. For example, 
when one is only interested in a histogram of the density of states, then one just has to continue until such a histogram doesn't change any more, within some desired accuracy, under enlargement of the tridiagonal Lanczos matrix.

For the results of sections 2 and 3 we did not need the whole spectrum, but only the lower part of it. The program we made for this has the following outline.

10 Set $i$ equal to 1 , and fix the value of $m_{1}$.

20 Generate a Lanczos matrix of order $m_{1}$, and compute all its eigenvalues in the interval of interest.

30 Consider any eigenvalue with a multiplicity larger than one as a " good » eigenvalue. Such eigenvalues have converged more than once.

40 For any simple eigenvalue, check whether it is also an eigenvalue of $\hat{T}_{2}^{\left(m_{1}\right)}$ If so, this eigenvalue is a «spurious » one.

If not, the eigenvalue is added to the list of " good " eigenvalues.

50 Check for each member of the list of isolated «good " eigenvalues whether it is also an eigenvalue of $T_{m_{i}-1}$. If so, this eigenvalue has converged.

If some eigenvalue has not converged yet, set $i$ to $i+1$, fix the value of $m_{1}$ and goto 20 . If all eigenvalues have converged goto 60.

60 Stop.

The choice of the values of $m_{1}(i=1,2$. ) depends mainly on the matrix being considered, i.e. on the distribution and separation of the eigenvalues. The closer the eigenvalues the bigger the Lanczos matrix one needs to separate them. To give an indication, for finding the lowest 78 eigenvalues of the dynamical matrix of order 1728 corresponding to the 3/2-approximant with a minimum separation of the eigenvalues of about $10^{-4}$ in the interval of interest, which is the interval $[0,1.1]$ of the total support $[0,3.2]$, the order of the Lanczos matrix required was between 800 and 1200 , depending on the wave vector $k$. In this case we have put $m$, equal to $800+(i-1) \times 50$.

With the Lanczos algorithm one cannot find the multiplicities of the eigenvalues. However, if it is known that eigenvalues occur with a multiplicity bigger than one, for example as a consequence of symmetry, then one can identify these multiple eigenvalues by slightly perturbing the matrix $H$. For the $k$-dependent dynamical matrix this can be done by slightly shifting the wave vector $\mathbf{k}$ from the high symmetry point. In principle, such a perturbation can also be applied to retrieve accidental degeneracies. In our application we had to find the eigenvalues of a large set of matrices, belonging to different $k$-vectors. It would therefore be very inefficient to check on potential degeneracies for each k-vector. However, knowing that the eigenvalues lie on a set of branch surfaces, which depend continuously on $\mathbf{k}$, one can easily find out when an eigenvalue is missing for a certain $\mathbf{k}$-vector. In our computations we did not find any accidental degeneracies. Nevertheless, it happened a few times, but only for the larger systems, that one of a group of very close eigenvalues was missing. It was easy to identify these missing eigenvalues, and in all cases the problem could be solved by rerunning the program for the matrix concerned, using a different starting vector for the Lanczos process. Obviously, the original starting vector had a very small projection on the eigenvector corresponding to the missing eigenvalue, so that it was rejected as a "spurious » eigenvalue. Also, the missing eigenvalues were only found among the highest eigenvalues in the interval of interest, and in our experience the problem can be avoided by enlarging the interval of interest somewhat. 


\section{Appendix B.}

\section{Brillouin zone integration method.}

Splitting the irreducible part of the Brillouin zone in equal tetrahedra, called simplices, six of them forming a cube, a quadratic interpolation of the branch surface within the simplex is given by :

$$
\omega_{s}(\mathbf{k})=a+\mathbf{b}^{\mathrm{T}} \mathbf{k}+\mathbf{k}^{\mathrm{T}} C \mathbf{k}
$$

where the ten free parameters of the scalar $a$, the three components vector $b$ and the symmetric $(3 \times 3)$-matrix $C$ are fixed by prescribing the values at the corners and the mid-edges of the simplices. A linear interpolation within the simplex can be written as:

$$
\omega_{s}(\mathbf{k})=a^{\prime}+\mathbf{b}^{\prime \top \mathrm{T}} \mathbf{k}
$$

where now only the values at the corners are needed to fix the four free parameters of $a^{\prime}$ and $\mathbf{b}^{\prime}$.

Methfessel [15] showed that with the quadratic fit the integration (8) can be performed analytically. However, we did not adopt this approach, but used, instead, the so-called hybrid method of MacDonald et al. [14]. In this method, each simplex is splitted up in smaller simplices of exactly the same form, and within each of these smaller simplices a linear interpolation is performed by using the quadratic fit, which holds for the large simplex, to determine the values at the corners of the small simplices. For the linear fit, evaluation of the integration (8) becomes quite easy.

On a computer one normally determines the density of states $D(\omega)$ at a discrete set of frequencies $\omega_{1}$, separated by a step length $\Delta \omega$. The value of $D\left(\omega_{1}\right)$ is then determined by adding the contributions from all simplices for which the frequency support contains $\omega_{1}$. However, using this method the contribution from a simplex gets lost whenever the frequency support of that simplex lies between two grid points in $\omega$-space. To avoid this problem, we determined the contribution from a simplex to $D\left(\omega_{1}\right)$ by evaluating the following integral :

$$
D\left(\omega_{1}\right)=\int_{\omega_{\min }}^{\omega_{\max }} \mathrm{d} \omega \frac{F_{l}(\omega) S(\omega)}{\left|\hat{\mathbf{n}} \cdot \nabla \omega_{s}(\mathbf{k})\right|}
$$

where $\omega_{\min }$ and $\omega_{\max }$ are, respectively, the minimal and the maximal frequency of the linearly interpolated branch surface within the simplex, $S(\omega)$ is the surface of constant $\omega$ inside the simplex, and the function $F,(\omega)$ is a weight function which we define as :

$$
F_{1}(\omega)=\left\{\begin{array}{cl}
\frac{\Delta \omega-\left|\omega-\omega_{1}\right|}{\Delta \omega} & \omega \in[\omega,-\Delta \omega, \omega,+\Delta \omega] \\
0 & \text { elsewhere }
\end{array}\right.
$$

so that a partial density from one simplex that falls in between two frequency grid points contributes equally to the total density at each of these grid points. Using this method all contributions are taken into account, and a very accurate normalization can be applied by dividing by the total volume of all simplices for all branches. Such a normalization is useful to compare the DOS for different structures.

Changing to another integration variable $t$, which is related to $\omega$ by:

$$
\omega(t)=\omega_{\min }+\left(\omega_{\max }-\omega_{\min }\right) t
$$


the integral (B3) can be rewritten as :

$$
D\left(\omega_{1}\right)=\frac{\left(\omega_{\max }-\omega_{\min }\right)}{\left|\hat{\mathbf{n}} \cdot \nabla \omega_{s}(\mathbf{k}) \lambda\right|} \int_{0}^{1} \mathrm{~d} t F_{1}(\omega(t)) S(\omega(t))
$$

where we have used the fact that $\left|\hat{\mathbf{n}} \cdot \nabla \omega_{s}(\mathbf{k})\right|$ is a constant as a consequence of the linearity of the interpolated branch surface. Another consequence of this linearity is that the surface of constant $\omega, S(t)$, depends quadratically on the parameter $t$. Let $\omega_{1}(i=1,4)$ be the four values taken by the linearly interpolated branch surface at the corners of the simplex, ordered in ascending order, so that $\omega_{1}=\omega_{\min }$ and $\omega_{4}=\omega_{\max }$. These four values are related to four values of $t, t_{1}(i=1,4)$, by the expression (B5) with $t_{1}=0$ and $t_{4}=1$. Depending on the possible degeneracies of the $\omega_{1}$ 's, the function $S(t)$ can have the following forms on the interval $t \in[0,1]$.

(i) If $0<t_{2}<t_{3}<1$

$$
S(t)= \begin{cases}a t^{2} & 0 \leqslant t<t_{2} \\ a t^{2}-b\left(t-t_{2}\right)^{2} & t_{2} \leqslant t \leqslant t_{3} \\ c(1-t)^{2} & t_{3}<t \leqslant 1\end{cases}
$$

where the positive constants $a, b$ and $c$ can be fixed by determination of $S\left(t_{2}\right)$ and $S\left(t_{3}\right)$.

(ii) If $0=t_{2}<t_{3}<1$

$$
S(t)= \begin{cases}a(1-t)^{2}-b\left(t_{3}-t\right)^{2} & 0 \leqslant t<t_{3} \\ a(1-t)^{2} & t_{3} \leqslant t \leqslant 1\end{cases}
$$

where $a, b$ can be fixed by calculating $S\left(t_{3}\right)$ and by using that $S(0)=0$.

(iii) If $0<t_{2}=t_{3}<1$

$$
S(t)= \begin{cases}a t^{2} & 0 \leqslant t<t_{2} \\ b(1-t)^{2} & t_{2} \leqslant t \leqslant 1\end{cases}
$$

where $a, b$ can be fixed by calculating $S\left(t_{2}\right)$.

(iv) If $0<t_{2}<t_{3}=1$

$$
S(t)= \begin{cases}a t^{2} & 0 \leqslant t<t_{2} \\ a t^{2}-b\left(t-t_{2}\right)^{2} & t_{2} \leqslant t \leqslant 1\end{cases}
$$

where $a, b$ can be fixed by calculating $S\left(t_{3}\right)$ and using that $S(0)=0$.

(v) If $0=t_{2}=t_{3}<1$ or $0<t_{2}=t_{3}=1$

$$
S(t)=a t(1-t) \quad 0 \leqslant t \leqslant 1
$$

where $a$ can be determined by using that $\int_{0}^{1} \mathrm{~d} t S(t)$ is equal to the volume of the simplex.

In the exceptional case that $\omega_{1}=\omega_{2}=\omega_{3}=\omega_{4}$ the contribution from the simplex is determined by

$$
D\left(\omega_{1}\right)=F_{1}\left(\omega_{1}\right) v
$$

where $v$ the volume of the simplex. 


\section{Acknowledgments.}

This work has been supported by the « Stichting voor Fundamenteel Onderzoek der Materie » (F.O.M.) with financial support of the « Nederlandse Organisatie voor Wetenschappelijk Onderzoek» (N.W.O.). We thank B. Polman for fruitful discussion about the Lanczos algorithm and for useful remarks about our description of the Lanczos algorithm without reorthogonalization in the Appendix A. Thanks are also expressed to Geert Rolf for establishing a parallel computer configuration which allowed us to perform the calculations within a reasonable amount of time.

\section{References}

[1] Los J., Janssen T. and Gahler F., J. Phys. France I 3 (1993) 107.

[2] JANSSEN T., Europhys. Lett. 14 (1991) 131.

[3] TANg L. H., Phys. Rev. Lett. 64 (1990) 2390.

[4] Los J. and JAnsSen T., J. Phys. Cond. Matter. 2 (1990) 9553.

[5] Конmoto M. and Banavar J. R., Phys. Rev. B 34 (1986) 563.

[6] Goldman A. I., Stassis C., de Boissieu M., Currat R., Janot C., Bellissent R., Mouddon H., Gayle F. W., Phys. Rev. B 45 (1992) 10280.

[7] Suck J. B., Bretscherand H., Guntherodt H. J., Z. Phys. B : Cond. Matter 68 (1987) 285.

[8] Quilichini M., Heger G., Hennion B., Lefebvre S., Quivy A., J. Phys. France 51 (1990) 1785.

[9] Haydock R., Heine V., Bullet D. and Kelly M. J., Solid State Physics 35 (Academic press, New York, 1980).

[10] Paige C. C., J. Inst. Math. Appl. 10 (1972) 373.

[11] Paige C. C., Lin. Algebra Appl. 34 (1980) 234.

[12] Cullum J. K. and Willoughby R. A., Lanczos algorithms for large symmetric eigenvalue computation (Birkhäuser, 1985).

[13] Parlett B. N. and Reid J. K., J. Num. Anal. 1 (1981) 135.

[14] MacDonald A. H., Vosko S. H. and Coleridge P. T., J. Phys. Cond. Matter 12 (1979) L 949.

[15] Methfessel L. H., J. Phys. Cond. Matter 16 (1983) 949.

[16] Los J., JANSSEN T. and GAHLER F., The phonon spectrum of the octagonal tiling, Int. J. Mod. Phys. B, in press (1993).

[17] LovesEY S. W., Theory of Neutron Scattering from Condensed Matter (Oxford University Press, 1985).

[18] Gianzotti P., Grosso G., Moroni S. and Pastori Parravinci G., Appl. Num. Math. 4 (1988) 273.

[19] Nex C. M. M., Solid State Physics 58 (Springer Series, New York, 1984). 